\title{
On the geometry of generalized Chaplygin systems
}

\author{
BY FRANS CANTRIJN \\ Department of Mathematical Physics and Astronomy, Ghent University \\ Krijgslaan 281, B-9000 Ghent, Belgium \\ e-mail: Frans.Cantrijn@rug.ac.be \\ JORGE CORTÉS † MANUEL DE LEÓN $\ddagger$ \\ AND DAVID MARTÍN DE DIEGO \\ Laboratory of Dynamical Systems, Mechanics and Control \\ Instituto de Matemáticas y Física Fundamental, CSIC \\ Serrano 123, 28006 Madrid, Spain \\ $\dagger e-m a i l:$ j.cortes@imaff.cfmac.csic.es $\ddagger e-m a i l$ : mdeleon@imaff.cfmac.csic.es \\ ๆe-mail: d.martin@imaff.cfmac.csic.es
}

October 22,2018

Short title: Chaplygin systems

2000 MS Classification: 37J60, 70Hxx

PACS: $02.40+\mathrm{m}, 03.20+\mathrm{i}$ 


\section{Abstract}

Some aspects of the geometry and the dynamics of generalized Chaplygin systems are investigated. First, two different but complementary approaches to the construction of the reduced dynamics are reviewed: a symplectic approach and an approach based on the theory of affine connections. Both are mutually compared and further completed. Next, a necessary and sufficient condition is derived for the existence of an invariant measure for the reduced dynamics of generalized Chaplygin systems of mechanical type. A simple example is then constructed of a generalized Chaplygin system which does not verify this condition, thereby answering in the negative a question raised by Koiller.

\section{Introduction}

In the past two decades, the rich developments in the field of Geometric Mechanics have led to a considerable progress in the study of the geometrical structure and of the dynamics of mechanical systems with nonholonomic constraints. Several concepts and techniques, familiar from the geometric approach to Lagrangian and Hamiltonian mechanics, have been succesfully adapted or extended to the framework of nonholonomic mechanics. Examples of this succesful "transfer of ideas" can be found, among others, in the symplectic and Poisson descriptions of constrained systems [5, 15, 21, 22, 26, 27, 39] and, in particular, in the study of nonholonomic systems with symmetry (reduction and reconstruction of the dynamics, stability of relative equilibria ...) where elements are being used from the theory of symplectic reduction and from the theory of principal connections and Ehresmann connections [5, 0, 10, 13, 20, 30]. Whereas most of the foregoing treatments are concerned with the autonomous case (i.e. time-invariant systems with time-independent constraints), the extension to time-dependent nonholonomic systems, using the geometric formalism of jet bundle theory, has also been treated by several authors: see e.g. 224, 32, 37] and references therein.

An important topic which is receiving growing attention in the literature, concerns the identification and characterization of a suitable notion of complete integrability of nonholonomic systems (see e.g. 22, 3, 6, 16, 17, 23, 41]). As is well known, an (unconstrained) Hamiltonian system on a $2 n$-dimensional phase space is called completely integrable if it admits $n$ independent integrals of motion in involution. It then follows from the Arnol'd-Liouville theorem that, when assuming compactness of the common level sets of these first integrals, the motion in phase space is quasiperiodic and consists of a winding on $n$-dimensional invariant tori (see e.g. [2], Chapter 4). For the integrability of a nonholonomic system with $k$ constraints one needs, in general, $2 n-k-1$ independent first integrals. It turns out, however, that for a nonholonomic system which admits an invariant measure, "only" $2 n-k-2$ first integrals are needed in order to reduce its integration to quadratures, and in such a case - again assuming compactness of the common level sets of the first integrals - the phase space trajectories of the system live on 2-dimensional invariant tori [2]. Several authors have studied the problem of the existence of invariant measures for some special classes of nonholonomic systems. For instance, Veselov and Veselova 41] have studied nonholonomic geodesic 
flows on Lie groups with a left-invariant metric and a right-invariant nonholonomic distribution (the so-called LR systems). Kozlov [23] has treated the analogous problem for left-invariant constraints. Their results have been very useful for finding new examples of completely integrable nonholonomic dynamical systems [16, 17, 41].

In the present paper we will mainly be concerned with a particular, but important class of nonholonomic systems: the so-called generalized Chaplygin systems. A system of generalized Chaplygin type is described by a principal connection on a principal fiber bundle $Q \longrightarrow Q / G$, whose horizontal distribution determines the constraint submanifold, and a $G$-invariant Lagrangian $L: T Q \longrightarrow \mathbb{R}$. Generalized Chaplygin systems exhibit a very nice geometric structure, which has been discussed extensively in the recent literature (see e.g. [7, 11, 13, 20, 27, 36, 43], and references therein). One of the peculiarities of these systems is that, after reduction, they take on the form of an unconstrained system, subject to an "external" force of a special type. In [36] it is shown that in the abelian Chaplygin case, the reduced equations can be rewritten in the form of a Hamiltonian system with respect to an almost symplectic structure. We will see that this also holds in the nonabelian case. In his pioneering paper on the reduction of nonholonomic systems with symmetry, Koiller touches the problem of the existence of an invariant measure for the reduced dynamics of generalized Chaplygin systems (see [20], Section 9). Based on several known examples of such systems which do admit an invariant measure, Koiller asks the question whether this property might perhaps hold in general. The main result of the present paper is the derivation of a necessary and sufficient condition for the existence of an invariant measure for the reduced dynamics of a generalized Chaplygin system whose Lagrangian is of pure kinetic energy type. This condition then enables us to give a negative answer to Koiller's question by constructing a simple counter example.

Clearly, there is still much work to be done in order to obtain a deeper and more complete understanding of the structure and behaviour of completely integrable nonholonomic systems. Any further progress into this matter will also be of importance for the study of perturbations of integrable nonholonomic systems. Concerning the particular case of generalized Chaplygin systems, it would be of interest, for instance, to identify general classes of systems which do admit an invariant measure, and to use their characterization to tackle the problem of complete integrability.

This paper is organized as follows. In Section 2, we first give a very brief review of some aspects of the theory of connections on principal bundles before introducing the notion of generalized Chaplygin system. Sections 3 and 4 are devoted to two alternative approaches to the description of generalized Chaplygin systems, with special emphasis on the reduction and reconstruction of their dynamics: a symplectic approach and an approach based on the use of affine connections. In Section 5, two examples are treated which exhibit a different behaviour in the reduction process. Section 6 then deals with the relation between the symplectic and the affine connection approach. The existence of an invariant measure for the reduced equations of generalized Chaplygin systems of mechanical type is studied in Section 7. The main result of the paper (Theorem 7.5) presents a necessary and sufficient condition for the existence of such an invariant measure, and an example is given of a system for which this condition is not satisfied. Finally, some particular results 
concerning the theory of affine connections are discussed in an Appendix.

Throughout this paper, we are working in the category of $C^{\infty}$-manifolds (with smooth maps, tensor fields, etc...). For convenience, we shall usually not make a notational distinction between a vector bundle over a manifold and the module of its smooth sections, i.e. if $\pi: F \rightarrow N$ denotes a vector bundle over a manifold $N$ (for instance a subbundle of $T N$ ), then $X \in F$ simply means that $X$ is a smooth section of $\pi$. The sole exception will be the sporadic use of the notation $\mathfrak{X}(N)$ for the module of vector fields on $N$. If $\mathcal{D}$ is distribution on a manifold $N$, then its annihilator is a codistribution on $N$ denoted by $\mathcal{D}^{o}$. Both $\mathcal{D}$ and $\mathcal{D}^{o}$ will also be identified with the corresponding vector subbundles of $T N$, resp. $T^{*} N$. Finally, the tangent map of a mapping $f$ between manifolds will be denoted interchangeably by $T f$ and $f_{*}$.

\section{Generalized Chaplygin systems}

In this section, we first briefly review the notion of a principal connection on a principal fibre bundle. For details we refer to [19]. Secondly, we consider a geometric framework for general nonholonomic mechanical systems and then introduce the concept of generalized Chaplygin systems.

\subsection{Principal connections}

Let $Q$ be the configuration manifold of a physical system and assume that there is a left action of a Lie group $G$ on $Q$ :

$$
\begin{aligned}
\Psi: G \times Q & \longrightarrow Q \\
(g, q) & \longmapsto \Psi(g, q)=\Psi_{g}(q)=g q .
\end{aligned}
$$

Note that we consider here left actions, which is the usual convention in mechanics, instead of the right ones, as considered for instance in [19]. The orbit through a point $q$ is $\operatorname{Orb}_{G}(q)=\{g q \mid g \in G\}$.

We denote by $\mathfrak{g}$ the Lie algebra of $G$. For any element $\xi \in \mathfrak{g}, \xi_{Q}$ will denote the corresponding infinitesimal generator of the group action on $Q$. Then,

$$
T_{q}\left(\operatorname{Orb}_{G}(q)\right)=\left\{\xi_{Q}(q) \mid \xi \in \mathfrak{g}\right\}
$$

Assuming that the action $\Psi$ is free and proper, we can endow the quotient space $Q / G=M$ with a manifold structure such that the canonical projection $\pi: Q \longrightarrow M$ is a surjective submersion. In the framework of the mechanics of (coupled) rigid bodies, for instance, the quotient manifold $M$ is commonly called the shape space of the system under consideration. We then have that $Q(M, G, \pi)$ is a principal bundle with bundle space $Q$, base space $M$, structure group $G$ and projection $\pi$. Note that the kernel of $\pi_{*}(=T \pi)$ consists of the vertical tangent vectors, i.e. the vectors tangent to the orbits of $G$ in $Q$. We shall denote the bundle of vertical vectors by $\mathcal{V}_{\pi}$, with $\left(\mathcal{V}_{\pi}\right)_{q}=T_{q}\left(\operatorname{Orb}_{G}(q)\right), q \in Q$.

A principal connection on $Q(M, G, \pi)$ can be defined as a distribution $\mathcal{H}$ on $Q$ satisfying:

(i) $T_{q} Q=\mathcal{H}_{q} \oplus\left(\mathcal{V}_{\pi}\right)_{q}, \forall q \in Q$; 
(ii) $\mathcal{H}_{g q}=T_{q} \Psi_{g}\left(\mathcal{H}_{q}\right)$, i.e. the distribution $\mathcal{H}$ is $G$-invariant;

(iii) $\mathcal{H}_{q}$ depends smoothly on $q$.

The subspace $\mathcal{H}_{q}$ of $T_{q} Q$ is called the horizontal subspace at $q$ determined by the connection. Alternatively, a principal connection can be characterized by a $\mathfrak{g}$-valued 1-form $\gamma$ on $Q$ satisfying the following conditions

(i) $\gamma\left(\xi_{Q}(q)\right)=\xi$ for all $\xi \in \mathfrak{g}$,

(ii) $\gamma\left(T \Psi_{g} X\right)=\operatorname{Ad}_{g}(\gamma(X))$ for all $X \in T Q$.

The horizontal subspace at $q$ is then given by $\mathcal{H}_{q}=\left\{v_{q} \in T_{q} Q \mid \gamma\left(v_{q}\right)=0\right\}$. A vector field $X$ on $Q$ is called horizontal if $X(q) \in \mathcal{H}_{q}$ at each point $q$.

Given a principal connection, we have that every vector $v \in T_{q} Q$ can be uniquely written as

$$
v=v_{1}+v_{2}
$$

with $v_{1} \in \mathcal{H}_{q}$ and $v_{2} \in\left(\mathcal{V}_{\pi}\right)_{q}$. We denote by $\mathbf{h}: T Q \longrightarrow \mathcal{H}$ and $\mathbf{v}: T Q \longrightarrow \mathcal{V}_{\pi}$ the corresponding horizontal and vertical projector, respectively. The horizontal lift of a vector field $Y$ on $M$ is the unique vector field $Y^{h}$ on $Q$ which is horizontal and projects onto $Y$.

The curvature $\Omega$ of the principal connection is the $\mathfrak{g}$-valued 2-form on $Q$ defined as follows: for each $q \in Q$ and $u, v \in T_{q} Q$

$$
\Omega(u, v)=d \gamma(\mathbf{h} u, \mathbf{h} v)=-\gamma\left(\left[U^{h}, V^{h}\right]_{q}\right)
$$

where $U^{h}$ and $V^{h}$ are the horizontal lifts of any two (local) vector fields $U$ and $V$ on $M$ for which $U^{h}(q)=\mathbf{h} u$ and $V^{h}(q)=\mathbf{h} v$, respectively. The curvature measures the lack of integrability of the horizontal distribution and plays a fundamental role in the theory of holonomy (see [19] for a comprehensive treatment).

\subsection{Nonholonomic mechanics and generalized Chaplygin systems}

A nonholonomic Lagrangian system consists of a Lagrangian $L$ defined on $T Q$, the tangent bundle of an $n$-dimensional configuration manifold $Q$, and constraints which determine a submanifold $\mathcal{D}$ of $T Q$. This means that the only allowable velocities are those belonging to $\mathcal{D}$. In case $\mathcal{D}$ is a vector subbundle of $T Q$, we are dealing with the case of linear constraints and if, in addition, this subbundle corresponds to an integrable distribution, we are reduced to the case of holonomic constraints. In the sequel, we will always assume that $\tau_{Q}(\mathcal{D})=Q$, where $\tau_{Q}: T Q \rightarrow Q$ is the tangent bundle projection.

Let $\left(q^{A}\right), A=1, \ldots, n$, be local coordinates on $Q$ and denote the induced bundle coordinates on $T Q$ by $\left(q^{A}, \dot{q}^{A}\right)$. In a local description, a constraint submanifold $\mathcal{D}$ of codimension $k$ can be defined by the vanishing of $k$ independent functions $\phi_{i}$ (the constraint functions). In the following, we shall 
only consider the case of linear constraints, such that the functions $\phi_{i}$ can be taken to be of the form $\phi_{i}(q, \dot{q})=\mu_{i A}(q) \dot{q}^{A}, i=1, \ldots, k$. Application of d'Alembert's principle, using the common notion of virtual displacement for the case of linear constraints, then leads to the constrained equations of motion

$$
\frac{d}{d t}\left(\frac{\partial L}{\partial \dot{q}^{A}}\right)-\frac{\partial L}{\partial q^{A}}=\lambda^{i} \mu_{i A}
$$

which, together with the constraint equations $\mu_{i A} \dot{q}^{A}=0(i=1, \ldots, k)$, determine the dynamics of the nonholonomic system. Here, the $\lambda^{i}$ are Lagrange multipliers to be determined. The right-hand side of equation (11) precisely represents the "reaction force" induced by the constraints.

We now describe the structure of a so-called generalized Chaplygin system [7, 20, 27]. The configuration manifold $Q$ of a generalized Chaplygin system is a principal $G$-bundle $\pi: Q \longrightarrow Q / G$, and the constraint submanifold $\mathcal{D}$ is given by the horizontal distribution $\mathcal{H}$ of a principal connection $\gamma$ on $\pi$. Furthermore, the system is described by a regular Lagrangian $L: T Q \longrightarrow \mathbb{R}$, which is $G$-invariant for the lifted action of $G$ on $T Q$. In this paper, we shall mainly restrict our attention to systems of mechanical type for which $L=T-V$, where $T: T Q \longrightarrow \mathbb{R}$ is the kinetic energy, corresponding to a Riemannian metric $g$ on $Q$, and $V: Q \longrightarrow \mathbb{R}$ is the potential energy. Whenever we add the word "mechanical" to the description of a system, we shall always be referring to this situation. For the case of a Chaplygin system we then suppose, in addition, that both the potential energy and the metric $g$ are $G$-invariant so that

$$
\mathcal{L}_{\xi_{Q}} V=0, \quad \mathcal{L}_{\xi_{Q}} g=0
$$

for all $\xi \in \mathfrak{g}$. In particular, it follows that all fundamental vector fields $\xi_{Q}$ are Killing vector fields. Typical problems in mechanics, such as the vertical and the inclined rolling disk, the nonholonomic free particle and the two wheeled carriage, can be interpreted as generalized Chaplygin systems in the above sense. Systems of that type also occur in many problems of robotic locomotion [18] and motions of microorganisms at low Reynolds number [35]. The dynamics of generalized Chaplygin systems can be described from a symplectic point of view, as we will show in the next section. However, for generalized Chaplygin systems of mechanical type there also exists a nice geometric description in terms of affine connections. This will be outlined in Section 4.

We conclude this section with some comments concerning terminology. Classically, a mechanical system with Lagrangian $L\left(q^{A}, \dot{q}^{A}\right), A=1, \ldots, n$, subject to $k$ linear nonholonomic constraints, is said to be of Chaplygin type if coordinates $\left(q^{a}, q^{\alpha}\right)$ can be found, with $a=1, \ldots, k$ and $\alpha=$ $k+1, \ldots, n$, such that the constraints can be written in the form $\dot{q}^{a}=B_{\alpha}^{a}\left(q^{k+1}, \ldots, q^{n}\right) \dot{q}^{\alpha}$ and such that $L$ does not depend on the coordinates $q^{a}$ (see e.g. 333). Such a system can be (locally) interpreted as a special case of the generalized Chaplygin systems introduced above, with $Q=\mathbb{R}^{n}$ and with an action defined by the abelian group $G=\mathbb{R}^{k}$ (cf. [20]). Koiller refers to the more general case, considered in the present paper, as "non-abelian Chaplygin systems". In the literature on nonholonomic systems with symmetry, these systems are also said to be of "principal" or "purely kinematical" type [7, 10, 20]. Finally, it should be emphasized that the generalized Chaplygin 
systems studied in [27] are still of a more general type than the ones we consider here in that they are defined on fibre bundles which need not be principal bundles.

\section{Symplectic approach}

As mentioned in the Introduction, considerable efforts have been made to adapt and extend several ideas and techniques from the geometric treatment of unconstrained problems to the study of systems with nonholonomic constraints. The subject has been approached from different points of view: a Lagrangian approach [0, 21, 34], a Hamiltonian approach [5, 15] and a formulation in terms of (almost-)Poisson structures 12, 22, 30, 39]. In this section, we start with a brief review of an interesting symplectic approach to nonholonomic dynamics, developed in [26, 27] which, in particular, is well suited for the treatment of nonholonomic systems with symmetry. We first outline this approach for general nonholonomic systems, and then turn to the case of generalized Chaplygin systems for the discussion of reduction and reconstruction of the constrained dynamics.

We start by fixing some notations. In terms of the tangent bundle coordinates $\left(q^{A}, \dot{q}^{A}\right)$, let us denote by $\Delta=\dot{q}^{A} \frac{\partial}{\partial \dot{q}^{A}}$ the dilation vector field on $T Q$ and by $S=d q^{A} \otimes \frac{\partial}{\partial \dot{q}^{A}}$ the canonical vertical endomorphism (see [25]). The action of $S$ on a 1 -form will be denoted by $S^{*}$. Then we can define the Poincaré-Cartan 1-form and 2-form, corresponding to a given Lagrangian $L$, by $\theta_{L}=S^{*} d L$ and $\omega_{L}=-d \theta_{L}$, respectively. We further have that $E_{L}=\Delta L-L$ represents the energy function of the system. If the Lagrangian $L$ is regular, which will always be tacitly assumed in the sequel, $\omega_{L}$ is symplectic and induces two isomorphisms of $C^{\infty}(T Q)$-modules:

$$
b_{L}: \mathfrak{X}(T Q) \longrightarrow \Omega^{1}(T Q), \quad \sharp_{L}: \Omega^{1}(T Q) \longrightarrow \mathfrak{X}(T Q),
$$

where $b_{L}(X)=i_{X} \omega_{L}$ and $\sharp_{L}=b_{L}^{-1}$. In the absence of constraints, the dynamics of the Lagrangian system, with Lagrangian $L$, is given by the (unique) solution $\Gamma_{L}$ of the equation $i_{\Gamma_{L}} \omega_{L}=d E_{L}$, i.e. $\Gamma_{L}=\sharp_{L}\left(d E_{L}\right)$. Indeed, $\Gamma_{L}$ is a second-order differential equation field (SODE for short) whose integral curves $\left(q^{A}(t), \dot{q}^{A}(t) \equiv \frac{d q^{A}}{d t}(t)\right)$ are determined by the solutions $q^{A}(t)$ of the Euler-Lagrange equations for $L$.

In the presence of nonholonomic constraints, the equations of motion must be modified in order to incorporate the constraints into the picture. Since we confine ourselves here to the case of linear constraints, the constraint submanifold $\mathcal{D}$ is a vector subbundle of $T Q$, determined by a (nonintegrable) distribution on $Q$ which we also denote by $\mathcal{D}$. In addition, we shall always assume that the constraints verify the so-called "admissibility condition" (see e.g. [27]), i.e. for all $x \in \mathcal{D}$

$$
\operatorname{dim}\left(T_{x} \mathcal{D}\right)^{o}=\operatorname{dim} S^{*}\left(\left(T_{x} \mathcal{D}\right)^{o}\right)
$$

where the annihilator of $T_{x} \mathcal{D}$ is taken in $T_{x}^{*} T Q$. Locally, $\mathcal{D}$ is described by equations of the form

$$
\mu_{i A}(q) \dot{q}^{A}=0, \quad i=1, \ldots k
$$


with $k=\operatorname{codim}(\mathcal{D})$. Next, we define a distribution $F$ on $T Q$ along $\mathcal{D}$, by prescribing its annihilator to be a subbundle of $T^{*} T Q_{\mid \mathcal{D}}$ which represents the bundle of reaction forces. More precisely, we set $F^{o}=S^{*}\left((T \mathcal{D})^{o}\right)$. The equations of motion for the nonholonomic system are then given by

$$
\left\{\begin{array}{l}
\left(i_{X} \omega_{L}-d E_{L}\right)_{\mid \mathcal{D}} \in F^{o}, \\
X_{\mid \mathcal{D}} \in T \mathcal{D} .
\end{array}\right.
$$

This system will have a unique solution $X$ provided the "compatibility condition" holds, i.e. $F^{\perp} \cap$ $T \mathcal{D}=0$, where $F^{\perp}=\sharp_{L}\left(F^{o}\right)$. For systems of mechanical type, with linear nonholonomic constraints and positive definite kinetic energy, this condition is always fulfilled. Indeed, putting

$$
C_{i j}=-\mu_{i A} W^{A B} \mu_{j B}, \quad i, j=1, \ldots k,
$$

where $\left(W^{A B}\right)$ is the inverse of the Hessian matrix $\left(\frac{\partial^{2} L}{\partial \dot{q}^{A} \partial \dot{q}^{B}}\right)$, compatibility locally translates into regularity of the matrix $\left(C_{i j}\right)$ (cf. [27], where the compatibility condition for a nonholonomic system was called the regularity condition). Under this condition, one can show that we have a direct sum decomposition of $T_{\mathcal{D}} T Q=T \mathcal{D} \oplus F^{\perp}$ and that the constrained dynamics $X$ is obtained by projecting the unconstrained Euler-Lagrange vector field $\Gamma_{L}$ (restricted to $\mathcal{D}$ ) onto $T \mathcal{D}$ with respect to this decomposition.

It should be pointed out that the solution $X$ of (2) satisfies automatically the SODE condition along $\mathcal{D}$, i.e. $S(X)_{\mid \mathcal{D}}=\Delta_{\mid \mathcal{D}}$. This implies that, in local coordinates, the integral curves of $X$ on $\mathcal{D}$ are of the form $\left(q^{A}(t), \dot{q}^{A}(t) \equiv \frac{d q^{A}}{d t}(t)\right)$, whereby the $q^{A}(t)$ are solutions of the system of differential equations (1), together with the constraint equations $\mu_{i A}(q) \dot{q}^{A}=0, i=1, \ldots, k$. The local coordinate expression for $X$ reads

$$
\begin{aligned}
X & =\dot{q}^{A} \frac{\partial}{\partial q^{A}} \\
& +W^{A B}\left(\frac{\partial L}{\partial q^{B}}-\frac{\partial p_{B}}{\partial q^{C}} \dot{q}^{C}+C^{i j} \frac{\partial \mu_{i D}}{\partial q^{C}} \mu_{j B} \dot{q}^{C} \dot{q}^{D}+W^{C D}\left(\frac{\partial L}{\partial q^{D}}-\dot{q}^{E} \frac{\partial p_{D}}{\partial q^{E}}\right) C^{i j} \mu_{j B} \mu_{i C}\right) \frac{\partial}{\partial \dot{q}^{A}},
\end{aligned}
$$

where $\left(C^{i j}\right)$ is the inverse of the matrix $\left(C_{i j}\right)$ introduced above and where, for ease of writing, we have put $p_{A}=\frac{\partial L}{\partial \dot{q}^{A}}, A=1, \ldots, n$.

Before proceeding, we recall that for nonholonomic Lagrangian systems with constraints which are linear (or, more general, homogeneous) in the velocities, the energy $E_{L}$ is a conserved quantity (see e.g. [10]). This, therefore, in particular applies to the generalized Chaplygin systems considered in this paper.

\subsection{Reduction}

The reduction theory of nonholonomic systems with symmetry, and related aspects, has become an intensive field of research 15, 0, 10, 11, 13, 22, 30, 34. Here we consider the special case of nonholonomic systems of generalized Chaplygin type.

The given data are (cf. Section 2): a principal $G$-bundle $\pi: Q \longrightarrow M=Q / G$, associated to a free and proper action $\Psi$ of $G$ on $Q$, a Lagrangian $L: T Q \longrightarrow \mathbb{R}$ which is $G$-invariant with respect to the 
lifted action on $T Q$, and linear nonholonomic constraints determined by the horizontal distribution (here denoted as $\mathcal{D}$ ) of a principal connection $\gamma$ on $\pi$. Taking into account the available symmetries, we can reduce the number of degrees of freedom of the problem. In the following, we review the various geometric concepts involved in the symplectic approach to this reduction process [10, 11, 13].

The symplectic action. Consider the lifted action of $G$ on $T Q$, i.e. $\hat{\Psi}: G \times T Q \longrightarrow T Q$ with $\hat{\Psi}\left(g, v_{q}\right)=T \Psi_{g}\left(v_{q}\right)\left(=\Psi_{g_{*}}\left(v_{q}\right)\right)$ for any $g \in G$ and $v_{q} \in T_{q} Q$. This action is also free and proper and, moreover, it is symplectic with respect to $\omega_{L}$. For any $\xi \in \mathfrak{g}$, the infinitesimal generators $\xi_{T Q}$ and $\xi_{Q}$ of $\hat{\Psi}$ and $\Psi$, respectively, are $\tau_{Q}$-related, i.e.

$$
\tau_{Q_{*}} \circ \xi_{T Q}=\xi_{Q} \circ \tau_{Q}
$$

Let us denote by $\rho: T Q \longrightarrow \overline{T Q}=T Q / G$ the natural projection. From the given assumptions it follows that the constraint submanifold $\mathcal{D}$, the energy $E_{L}$ and the vector subbundle $F$ are $G$ invariant. The induced action of $G$ on $\mathcal{D}$, i.e. the restriction of $\hat{\Psi}$ to $G \times \mathcal{D}$, is still free and proper and we can regard the orbit space $\overline{\mathcal{D}}=\mathcal{D} / G$ as a submanifold of $T Q / G$. Note that there exists a natural identification $\mathcal{D} \cong Q \times{ }_{Q / G} T(Q / G)$ as principal $G$-bundles over $T(Q / G)$. The isomorphism is obtained by mapping $v_{q} \in \mathcal{D}$ onto $\left(q, \pi_{*}\left(v_{q}\right)\right)$. It then follows that $\mathcal{D} / G$ can be naturally identified with $T(Q / G)$ and we have, in particular,

$$
\rho_{\mid \mathcal{D}}=\pi_{* \mid \mathcal{D}}
$$

Henceforth, the restriction of $\rho$ to $\mathcal{D}$ will also be simply denoted by $\rho$.

The connection. A direct computation shows that along $\mathcal{D}$ we have that $\mathcal{V}_{\rho} \cap F=0$, where $\mathcal{V}_{\rho}$ denotes the subbundle of $T T Q$ which is vertical with respect to the projection $\rho$, i.e. $\mathcal{V}_{\rho}=\operatorname{Ker} T \rho$. Observing that $\mathcal{V}_{\rho \mid \mathcal{D}} \subset T \mathcal{D}$, it then easily follows that

$$
T \mathcal{D}=(F \cap T \mathcal{D}) \oplus \mathcal{V}_{\rho \mid \mathcal{D}}
$$

Since $\mathcal{U}=F \cap T \mathcal{D}$ is $G$-invariant, the above decomposition of $T \mathcal{D}$ defines a principal connection $\Upsilon$ on the principal $G$-bundle $\rho: \mathcal{D} \longrightarrow \overline{\mathcal{D}}=\mathcal{D} / G$, with horizontal subspace $\mathcal{U}_{x}$ at each $x \in \mathcal{D}$. Moreover, we have that the dynamics $X$ of the generalized Chaplygin system, determined by (2), belongs to $\mathcal{U}$. We shall denote the projection of the bundle $\mathcal{U}$ under $T \rho\left(=\rho_{*}\right)$ by $\overline{\mathcal{U}}$.

We can summarize the situation in the following diagram:

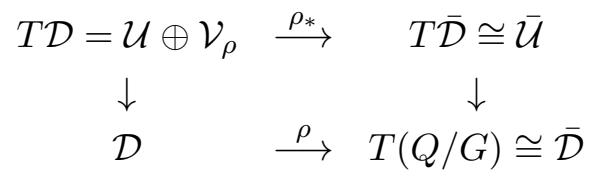

The principal connection $\Upsilon$ is obviously related to the original connection $\gamma$ of the Chaplygin system. Indeed, take $w \in T_{v_{q}} \mathcal{D}$ and consider $\tau_{Q_{*}} w \in T_{q} Q$. Then, we can write

$$
\tau_{Q_{*}} w=\left(\tau_{Q_{*}} w-\left(\gamma\left(\tau_{Q_{*}} w\right)\right)_{Q}(q)\right)+\left(\gamma\left(\tau_{Q_{*}} w\right)\right)_{Q}(q),
$$


where $\tau_{Q_{*}} w-\left(\gamma\left(\tau_{Q_{*}} w\right)\right)_{Q}(q) \in \mathcal{D}_{q}$ and $\left(\gamma\left(\tau_{Q_{*}} w\right)\right)_{Q}(q) \in\left(\mathcal{V}_{\pi}\right)_{q}$. Putting $\gamma\left(\tau_{Q_{*}} w\right)=\xi \in \mathfrak{g}$, a direct computation shows that $w-\xi_{T Q}\left(v_{q}\right) \in \mathcal{U}$ and, consequently, $w=\left(w-\xi_{T Q}\left(v_{q}\right)\right)+\xi_{T Q}\left(v_{q}\right)$ is the $\left(\mathcal{U}, \mathcal{V}_{\rho}\right)$ decomposition of $w$. Herewith we have proved the following property.

Proposition 3.1. The connection 1-forms $\Upsilon$ and $\gamma$ are related by $\Upsilon=\tau_{Q}^{*} \gamma$, i.e. $\Upsilon_{v_{q}}(w)=$ $\gamma_{q}\left(\tau_{Q_{*}} w\right)$ for any $v_{q} \in \mathcal{D}$ and $w \in T_{v_{q}} \mathcal{D}$.

Let us denote the horizontal projectors, associated to $\gamma$, resp. $\Upsilon$, by $\mathbf{h}_{\gamma}: T Q \longrightarrow \mathcal{D}(\subset T Q)$, resp. $\mathbf{h}_{\Upsilon}: T \mathcal{D} \longrightarrow \mathcal{U}(\subset T \mathcal{D})$. Likewise, the vertical projectors onto $\mathcal{V}_{\pi}$, resp. $\mathcal{V}_{\rho}$, will be denoted by $\mathbf{v}_{\gamma}$, resp. $\mathbf{v} \boldsymbol{\Upsilon}$. In order not to further overload the notations, we will use the same superscript ${ }^{h}$ for the horizontal lifts of vectors (vector fields) with respect to either $\gamma$ or $\Upsilon$; in principle it should always be clear from the context which horizontal lift operation is being used.

We now have that

$$
\tau_{Q_{* \mid T \mathcal{D}}} \circ \mathbf{h}_{\Upsilon}=\mathbf{h}_{\gamma} \circ \tau_{Q_{* \mid T \mathcal{D}}}
$$

i.e. the following diagram is commutative:

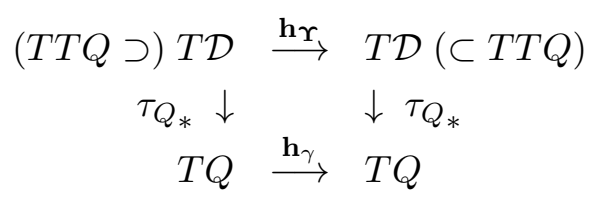

Indeed, taking into account Proposition 3.1 we see that for any $w \in T_{v_{q}} \mathcal{D}, \gamma_{q}\left(\tau_{Q_{*}} \mathbf{h}_{\Upsilon}(w)\right)=$ $\Upsilon_{v_{q}}\left(\mathbf{h}_{\Upsilon}(w)\right)=0$ and, hence, $\tau_{Q_{*}} \mathbf{h}_{\Upsilon}(w)$ is horizontal with respect to $\gamma$, i.e. $\tau_{Q_{*}} \mathbf{h}_{\Upsilon}(w) \in \mathcal{D}_{q}$. By definition we also have $\mathbf{h}_{\gamma}\left(\tau_{Q_{*}} w\right) \in \mathcal{D}_{q}$. Using the fact that $\pi \circ \tau_{Q}=\tau_{Q / G} \circ \pi_{*}$ we obtain:

$$
\pi_{*}\left(\mathbf{h}_{\gamma}\left(\tau_{Q_{*}} w\right)\right)=\pi_{*} \tau_{Q_{*}} w=\tau_{Q / G_{*}}\left(\pi_{*}\right)_{*} w=\tau_{Q / G_{*}} \rho_{*} w
$$

where the last equation follows from (41). Similarly, we have:

$$
\pi_{*}\left(\tau_{Q_{*}}\left(\mathbf{h}_{\Upsilon} w\right)\right)=\tau_{Q / G_{*}}\left(\pi_{*}\right)_{*}\left(\mathbf{h}_{\Upsilon} w\right)=\tau_{Q / G_{*}} \rho_{*}\left(\mathbf{h}_{\Upsilon} w\right)=\tau_{Q / G_{*}} \rho_{*} w
$$

We thus see that the $\gamma$-horizontal tangent vectors at $q, \tau_{Q_{*}}\left(\mathbf{h}_{\Upsilon} w\right)$ and $\mathbf{h}_{\gamma}\left(\tau_{Q_{*}} w\right)$, have the same projection under $\pi_{*}$ and, therefore, they are equal. This completes the proof of (5)). Denoting the curvature tensors of the principal connections $\gamma$ and $\Upsilon$ by $\Omega^{\gamma}$ and $\Omega^{\Upsilon}$, respectively, one can easily deduce from Proposition 3.1 and (5) the relation

$$
\Omega^{\Upsilon}=\tau_{Q}^{*} \Omega^{\gamma}
$$

The 1-form. Denote by $\theta^{\prime}$ the pullback to $\mathcal{D}$ of the Poincaré-Cartan 1-form $\theta_{L}$, i.e. $\theta^{\prime}=j_{\mathcal{D}}^{*} \theta_{L}$, where $j_{\mathcal{D}}: \mathcal{D} \hookrightarrow T Q$ is the canonical inclusion. By means of the solution $X$ of (2) we can construct a 1-form $\alpha_{X}$ on $\mathcal{D}$ as follows:

$$
\alpha_{X}=i_{X}\left(\mathbf{h}_{\Upsilon}{ }^{*} d \theta^{\prime}-d \mathbf{h}_{\Upsilon}{ }^{*} \theta^{\prime}\right),
$$

with the usual convention that, for an arbitrary $p$-form $\beta$ on $\mathcal{D}, \mathbf{h}_{\boldsymbol{\Upsilon}}{ }^{*} \beta$ is the $p$-form defined by $\mathbf{h}_{\Upsilon}{ }^{*} \beta\left(X_{1}, \ldots, X_{p}\right)=\beta\left(\mathbf{h}_{\Upsilon}\left(X_{1}\right), \ldots, \mathbf{h}_{\Upsilon}\left(X_{p}\right)\right)$. 
The Lagrangian. The Lagrangian $L$ of the given mechanical system induces a Lagrangian $L^{*}$ : $T(Q / G) \longrightarrow \mathbb{R}$ on the quotient space $\overline{\mathcal{D}} \cong T(Q / G)$, given by $L^{*}\left(\bar{q}, v_{\bar{q}}\right)=L\left(q, v_{q}^{h}\right)$ for any $q \in$ $\pi^{-1}(\bar{q})$, and where $v_{q}^{h}$ denotes the $\gamma$-horizontal lift of $v_{\bar{q}}$ at $q$. This is well-defined because of the $G$-invariance of $L$. Moreover, one can show that $L^{*}$ is a regular Lagrangian on $T(Q / G)$ (cf. [27]).

Now, we are in a position to state the following reduction result (see [11, 20]).

Proposition 3.2. The dynamics $X$ of the generalized Chaplygin system projects onto $\overline{\mathcal{D}}$, and its projection $\bar{X}$ is determined by the equation

$$
i_{\bar{X}} \omega_{L^{*}}=d E_{L^{*}}+\overline{\alpha_{X}},
$$

where $\overline{\alpha_{X}}$ is the projection of the 1-form $\alpha_{X}$, defined by (1). Moreover, we have that $i_{\bar{X}} \overline{\alpha_{X}}=0$.

REMARK 3.3. The sign of $\overline{\alpha_{X}}$ in equation (8) differs from the one in the corresponding expression derived in [11], where the discussion took place in a more general symplectic framework with an exact symplectic structure $\omega=d \theta$ (whereas here we have $\omega_{L}=-d \theta_{L}$ ). The signs would agree if we would have defined $\theta^{\prime}$ as $-j_{\mathcal{D}}^{*} \theta_{L}$.

It can be easily verified that the form $\overline{\alpha_{X}}$ is a semi-basic 1-form on $\overline{\mathcal{D}}$ (see also Section 6), from which it then follows that the vector field $\bar{X}$, defined by $(\mathbb{8})$, is a SODE. Moreover, one can show that not only the contraction of $\overline{\alpha_{X}}$ with $\bar{X}$ vanishes, but that, more generally, $i_{Y} \overline{\alpha_{X}}=0$ for any SODE $Y$ on $\overline{\mathcal{D}}$. We thus see that a generalized Chaplygin system reduces to an unconstrained mechanical system, with an external nonconservative force of "gyroscopic" type, which is geometrically represented by the 1 -form $\overline{\alpha_{X}}$ (see also [20, 33]). The "gyroscopic" character of this force is also in agreement with the fact that the projected energy function $E_{L^{*}}$ is a conserved quantity of the reduced dynamics. But there is more to be said about it.

Proposition 3.4. The 2-form $\Sigma=\mathbf{h}_{\Upsilon}{ }^{*} d \theta^{\prime}-d \mathbf{h}_{\Upsilon}{ }^{*} \theta^{\prime}$ on $\mathcal{D}$ projects onto a 2-form $\bar{\Sigma}$ on $\overline{\mathcal{D}}$ and the 1-form $\overline{\alpha_{X}}$ satisfies

$$
\overline{\alpha_{X}}=i_{\bar{X}} \bar{\Sigma}
$$

Proof. Let $\xi_{\mathcal{D}}$ be the fundamental vector field of the $G$-action on $\mathcal{D}$, induced by an arbitrary element $\xi \in \mathfrak{g}$. We must prove that $\xi_{\mathcal{D}}$ belongs to the characteristic distribution of the 2-form $\Sigma$. First, we have that

$$
i_{\xi_{\mathcal{D}}} \Sigma=-i_{\xi_{\mathcal{D}}} d \mathbf{h} \Upsilon^{*} \theta^{\prime}
$$

For any vector field $Y$ on $\mathcal{D}, i_{\xi_{\mathcal{D}}} d \mathbf{h}_{\Upsilon}{ }^{*} \theta^{\prime}(Y)=\xi_{\mathcal{D}}\left(\theta^{\prime}\left(\mathbf{h}_{\Upsilon} Y\right)\right)-\theta^{\prime}\left(\mathbf{h}_{\Upsilon}\left[\xi_{\mathcal{D}}, Y\right]\right)$. Now, if $Y$ is vertical, we readily see that $i_{\xi_{\mathcal{D}}} d \mathbf{h}_{\Upsilon}{ }^{*} \theta^{\prime}(Y)=0$. If $Y$ is horizontal, we have

$$
i_{\xi_{\mathcal{D}}} d \mathbf{h}_{\Upsilon}{ }^{*} \theta^{\prime}(Y)=\mathcal{L}_{\xi_{\mathcal{D}}} \theta^{\prime}(Y)=0,
$$

because of the $G$-invariance of $\theta^{\prime}$. It therefore remains to prove that $i_{\xi_{\mathcal{D}}} d \Sigma=0$. For any two vector fields $Y, Z$ we have that

$$
\begin{aligned}
i_{\xi_{\mathcal{D}}} d \Sigma(Y, Z) & =\left(i_{\xi_{\mathcal{D}}} d \mathbf{h}_{\Upsilon}{ }^{*} d \theta^{\prime}\right)(Y, Z) \\
& =\xi_{\mathcal{D}}\left(\mathbf{h}_{\Upsilon^{*}} d \theta^{\prime}\right)(Y, Z)-\mathbf{h}_{\Upsilon}{ }^{*} d \theta^{\prime}\left(\left[\xi_{\mathcal{D}}, Y\right], Z\right)+\mathbf{h}_{\Upsilon}{ }^{*} d \theta^{\prime}\left(\left[\xi_{\mathcal{D}}, Z\right], Y\right)
\end{aligned}
$$


If at least one of the vector fields $Y$ and $Z$ is vertical, then $i_{\xi_{\mathcal{D}}} d \Sigma(Y, Z)=0$. Taking $Y$ and $Z$ both horizontal, we find, taking into account the $G$-invariance of $d \theta^{\prime}$ and $\xi_{\mathcal{D}} \in \mathfrak{X}(\mathcal{D})$,

$$
i_{\xi_{\mathcal{D}}} d \Sigma(Y, Z)=\xi_{\mathcal{D}}\left(d \theta^{\prime}(Y, Z)\right)-d \theta^{\prime}\left(\left[\xi_{\mathcal{D}}, Y\right], Z\right)-d \theta^{\prime}\left(Y,\left[\xi_{\mathcal{D}}, Z\right]\right)=\left(\mathcal{L}_{\xi_{\mathcal{D}}} d \theta^{\prime}\right)(Y, Z)=0 .
$$

The last part of the proposition now immediately follows from (7) and the projectability of $X$.

$Q E D$

Consider a local trivialization $U \times G$ of $\pi$, with coordinates $\left(q^{a}, g^{i}\right)$, where $i=1, \ldots k=\operatorname{dim} G$ and $a=1, \ldots, n-k$. Choosing a basis $e_{i}(i=1, \ldots, k)$ of the Lie algebra $\mathfrak{g}$, and using the left trivialization $T G \cong G \times \mathfrak{g}$, a tangent vector $v \in T_{(x, g)}(U \times G) \cong T_{x} U \times \mathfrak{g}$ can be represented by a pair $(w, \xi)$, whereby $w \in T_{x} U$ and $\xi=\xi^{i} e_{i} \in \mathfrak{g}$. In terms of the coordinates $\left(q^{a}, g^{i}, \dot{q}^{a}, \xi^{i}\right)$ on $T(U \times G)$ the $G$-invariant Lagrangian can then be written as

$$
L=\ell\left(q^{a}, \dot{q}^{a}, \xi^{i}\right)
$$

Strictly speaking, $\ell$ represents the reduction of $L$ to $T Q / G$. With respect to the given local trivialization, we further denote the connection coefficients of the given principal connection $\gamma$ by $\Gamma_{a}^{i}=\Gamma_{a}^{i}\left(q^{1}, \ldots, q^{n-k}\right)$, and the constraints then take the form $\xi^{i}=-\Gamma_{a}^{i} \dot{q}^{a}$. In particular, it follows that the reduced Lagrangian $L^{*}$ is given by $L^{*}\left(q^{a}, \dot{q}^{a}\right)=\ell\left(q^{a}, \dot{q}^{a},-\Gamma_{b}^{i} \dot{q}^{b}\right)$.

With all the above one can now derive the following coordinate expression for the reduced dynamics (see also [0, 20]):

$$
\bar{X}=\dot{q}^{a} \frac{\partial}{\partial q^{a}}+\hat{W}^{a b}\left(\frac{\partial L^{*}}{\partial q^{b}}-\dot{q}^{c} \frac{\partial \hat{p}_{b}}{\partial q^{c}}-\alpha_{b}\right) \frac{\partial}{\partial \dot{q}^{a}},
$$

where $\left(\hat{W}^{a b}\right)$ is the inverse of the Hessian matrix $\left(\frac{\partial^{2} L^{*}}{\partial \dot{q}^{a} \partial \dot{q}^{b}}\right), \hat{p}_{a}=\frac{\partial L^{*}}{\partial \dot{q}^{a}}$, and $\alpha_{b} d q^{b}$ is the local expression for the gyroscopic 1 -form $\overline{\alpha_{X}}$. The $\alpha_{b}$ are explicitly given by

$$
\alpha_{b}=-\left(\frac{\partial \ell}{\partial \xi^{i}}\right)^{*}\left(\frac{\partial \Gamma_{c}^{i}}{\partial q^{b}}-\frac{\partial \Gamma_{b}^{i}}{\partial q^{c}}-c_{j k}^{i} \Gamma_{b}^{j} \Gamma_{c}^{k}\right) \dot{q}^{c},
$$

where the ${ }^{*}$ on the right-hand side indicates that, after computing the derivative of $\ell$ with respect to $\xi^{i}$, one replaces the $\xi^{j}$ everywhere by $-\Gamma_{a}^{j} \dot{q}^{a}$. The constants $c_{j k}^{i}$ appearing in the last term on the right-hand side are the structure constants of $\mathfrak{g}$ with respect to the chosen basis, i.e. $\left[e_{j}, e_{k}\right]=c_{j k}^{i} e_{i}$. Note in passing that the expressions $\frac{\partial \Gamma_{c}^{i}}{\partial q^{b}}-\frac{\partial \Gamma_{b}^{i}}{\partial q^{c}}-c_{j k}^{i} \Gamma_{b}^{j} \Gamma_{c}^{k}$ are the coefficients of the curvature of $\gamma$ in local form.

\subsection{Reconstruction}

A natural problem related to the reduction of mechanical systems with symmetry concerns the reverse procedure: once the solutions of the reduced dynamics have been obtained, how can one recover from it the solutions of the original system. This is called the "reconstruction problem" of the dynamics. This problem is intimately related to the concepts of geometric and dynamic phase, which play an important role in various aspects of mechanics [31] and in the study of locomotion systems (for example, in the generation of net motion by cyclic changes in shape space [18, 35]). 
In the case of a generalized Chaplygin system, the reconstruction of the dynamics on $\mathcal{D}$ from the reduced dynamics on $\overline{\mathcal{D}}$ proceeds as follows 13. Suppose that the flow of the reduced system $\bar{X}$ is known. Let $\bar{c}(t)$ be the integral curve of $\bar{X}$ starting at a given point $\bar{x} \in \overline{\mathcal{D}}$, and fix $x \in \rho^{-1}(\bar{x})$. We want to find the integral curve $c(t)$ of $X$ with initial point $x$ and projecting onto $\bar{c}(t)$, i.e. $\rho(c(t))=\bar{c}(t)$. But this is precisely the horizontal lift through $x$ of $\bar{c}(t)$, with respect to the principal connection $\Upsilon$. We recall here the proof of this simple fact (see also [13]).

Proposition 3.5. The integral curve $c(t)$ of the generalized Chaplygin system, starting at $x \in \mathcal{D}$, is the horizontal lift with respect to the principal connection $\Upsilon$ of the integral curve $\bar{c}(t)$ of the reduced system starting at $\bar{x}=\rho(x)$.

Proof. Let $d(t)$ denote the horizontal lift of $\bar{c}(t)$, starting at $x$ (say, at $t=0$ ). Therefore, $\dot{d}(t) \in$ $\mathcal{D}$ and $\rho(d(t))=\bar{c}(t)$ for each $t$, and $d(0)=x$. Since $X$ and $\bar{X}$ are $\rho$-related, we have that $T \rho(X(d(t)))=\bar{X}(\rho(d(t)))=\bar{X}(\bar{c}(t))=T \rho(\dot{d}(t))$. Hence, $\dot{d}(t)-X(d(t))$ is vertical for each $t$. On the other hand, it is also horizontal, since $X \in \mathcal{U}$. Therefore, we deduce that $\dot{d}(t)=X(d(t))$.

Summarizing: in the symplectic formalism, the reconstruction simply consists of a horizontal lift operation with respect to the induced connection $\Upsilon$ living on $\mathcal{D}$.

\section{Affine connection approach}

In this section we will describe the reduction and reconstruction problem for generalized Chaplygin systems of mechanical type, from a different point of view, namely in terms of the theory of affine connections. First, we recall some general results which apply to any kind of nonholonomic system of mechanical type.

Let $Q$ be the configuration space of a mechanical system with Lagrangian of the form

$$
L(v)=\frac{1}{2} g(v, v)-V \circ \tau_{Q}(v), v \in T_{x} Q,
$$

where $g$ is a Riemannian metric on $Q$ and $V: Q \longrightarrow \mathbb{R}$ is the potential energy function. We denote by $\nabla^{g}$ the (covariant derivative operator of the) Levi-Civita connection associated to the metric $g$. It is well-known that a curve $c: I \longrightarrow Q$ is a solution of the Euler-Lagrange equations for the (unconstrained) Lagrangian if and only if

$$
\nabla_{\dot{c}(t)}^{g} \dot{c}(t)=-\operatorname{grad} V(c(t))
$$

where the gradient is also considered with respect to the metric $g$.

For nonholonomic systems there is a similar description. Let $\mathcal{D}$ again denote a (nonintegrable) distribution on $Q$, describing some linear nonholonomic constraints. The second-order differential equations (11) for the mechanical system with Lagrangian $L$ and constraints $\mathcal{D}$, can be written intrinsically as

$$
\nabla_{\dot{c}(t)}^{g} \dot{c}(t)+\operatorname{grad} V(c(t)) \in \mathcal{D}_{\dot{c}(t)}^{\perp}, \quad \dot{c}(t) \in \mathcal{D}_{c(t)},
$$


where $\mathcal{D}^{\perp}$ here denotes the $g$-orthogonal complement to $\mathcal{D}$ (see e.g. [6, 28, 38, 40]).

Alternatively, if we denote by

$$
\mathcal{P}: T Q \longrightarrow \mathcal{D}, \quad \mathcal{Q}: T Q \longrightarrow \mathcal{D}^{\perp}
$$

the complementary $g$-orthogonal projectors, we can define an affine connection

$$
\bar{\nabla}_{X} Y=\nabla_{X}^{g} Y+\left(\nabla_{X}^{g} \mathcal{Q}\right)(Y),
$$

such that the nonholonomic equations of motion can be rewritten as

$$
\bar{\nabla}_{\dot{c}(t)} \dot{c}(t)=-\mathcal{P}(\operatorname{grad} V(c(t)))
$$

and where we select the initial velocity in $\mathcal{D}$ (cf. [28]).

In what follows, we shall restrict our attention to Lagrangians of "pure kinetic energy type", i.e. we assume $V=0$. The reason for doing this is twofold. First, it makes the geometric picture more clear and tractable, in that the equations of motion for the nonholonomic mechanical system can then be seen as the geodesic equations of an affine connection. Secondly, the extention to systems with a nontrivial potential energy function is rather straightforward but, at least for those aspects of nonholonomic dynamics that are of interest to us here, it does not really tell us anything new.

It can be easily deduced from its definition that the connection $\bar{\nabla}$ restricts to $\mathcal{D}$, that is,

$$
\bar{\nabla}_{X} Y \in \mathcal{D}
$$

for all $Y \in \mathcal{D}$ and $X \in \mathfrak{X}(Q)$. This kind of affine connections, which restrict to a given distribution, have been studied in [28]. In particular, such a behaviour implies that the distribution $\mathcal{D}$ is geodesically invariant, that is, for every geodesic $c(t)$ of $\bar{\nabla}$ starting from a point in $\mathcal{D}, \dot{c}(0) \in$ $\mathcal{D}_{c(0)}$, we have that $\dot{c}(t) \in \mathcal{D}_{c(t)}$. In [28], a nice property is derived which characterizes geodesically invariant distributions in terms of the so-called symmetric product of vector fields, which is defined as $\langle X: Y\rangle=\bar{\nabla}_{X} Y+\bar{\nabla}_{Y} X$. This property asserts that $\mathcal{D}$ is geodesically invariant if and only if we have that $\langle X: Y\rangle \in \mathcal{D}, \forall X, Y \in \mathcal{D}$. Note in passing that the symmetric product of vector fields is a differential geometric concept with important applications to control theory, first introduced in [29]. For instance, it plays a fundamental role in the controllability analysis of mechanical control systems [9, 14, 29], in the description of the evolution of these systems when starting from rest [8], and in the design of motion planning control algorithms [9].

Proposition 4.1. For all $Z \in \mathfrak{X}(Q)$ and $X, Y \in \mathcal{D}$ we have that

$$
Z(g(X, Y))=g\left(\bar{\nabla}_{Z} X, Y\right)+g\left(X, \bar{\nabla}_{Z} Y\right) .
$$

Proof. In view of the definition of $\bar{\nabla}$, we have that

$$
\begin{aligned}
g\left(\bar{\nabla}_{Z} X, Y\right)+g\left(X, \bar{\nabla}_{Z} Y\right) & =g\left(\nabla_{Z}^{g} X, Y\right)+g\left(X, \nabla_{Z}^{g} Y\right)+g\left(\left(\nabla_{Z}^{g} \mathcal{Q}\right)(X), Y\right)+g\left(X,\left(\nabla_{Z}^{g} \mathcal{Q}\right)(Y)\right) \\
& =Z(g(X, Y))
\end{aligned}
$$


since $\left(\nabla_{Z}^{g} \mathcal{Q}\right)(X),\left(\nabla_{Z}^{g} \mathcal{Q}\right)(Y) \in \mathcal{D}^{\perp}$ (see Proposition 6.1 in 28$]$ ).

We derive from this proposition that the connection $\bar{\nabla}$ also has the following property: parallel transport is an isometry along the distribution $\mathcal{D}$.

A direct computation shows that the torsion of $\bar{\nabla}$ is the skew-symmetric $(1,2)$-tensor field

$$
\bar{T}(X, Y)=\left(\nabla_{X}^{g} \mathcal{Q}\right)(Y)-\left(\nabla_{Y}^{g} \mathcal{Q}\right)(X) .
$$

Observe that if $X, Y \in \mathcal{D}$ then $\bar{T}(X, Y) \in \mathcal{D}^{\perp}$.

\subsection{Reduction}

We now return to the case of generalized Chaplygin systems of mechanical type, but again assuming, for simplicity, that $L$ only consists of a kinetic energy part (i.e. $V=0$ ). From the above we then know that the equations of motion of the system can be written as

$$
\bar{\nabla}_{\dot{c}(t)} \dot{c}(t)=0, \quad \dot{c}(0) \in \mathcal{D} .
$$

The following is mainly inspired on the reduction process of generalized (or non-abelian) Chaplygin systems as described in [20].

Let us define a metric $\tilde{g}$ on the base manifold $M(=Q / G)$ as follows

$$
\tilde{g}_{x}\left(u_{x}, v_{x}\right)=g_{q}\left(U_{q}, V_{q}\right), \quad x \in M, u_{x}, v_{x} \in T_{x} M,
$$

where $q \in \pi^{-1}(x)$ and $U_{q}, V_{q}$ are horizontal vectors which project under $\pi$ onto $u_{x}$ and $v_{x}$, respectively. From the $G$-invariance of $g$ we deduce that the right-hand side is independent of the chosen point $q$ in the fibre $\pi^{-1}(x)$ and, hence, $\tilde{g}$ is well defined.

Proposition 4.2. We have that for all $X, Y \in \mathfrak{X}(M)$ and $\xi \in \mathfrak{g}$

$$
\mathcal{L}_{\xi_{Q}}\left(\bar{\nabla}_{X^{h}} Y^{h}\right)=0
$$

Proof. Since $\xi_{Q}$ is a Killing vector field, it follows from Proposition VI. 2.2 in 19 that

$$
\mathcal{L}_{\xi_{Q}} \nabla_{X^{h}}^{g} Y^{h}=\nabla_{\left[\xi_{Q}, X^{h}\right]}^{g} Y^{h}+\nabla_{X^{h}}^{g}\left[\xi_{Q}, Y^{h}\right]=0
$$

because $Y^{h}$ and $X^{h}$ are projectable. Therefore, we only need to prove that

$$
\mathcal{L}_{\xi_{Q}}\left[\left(\nabla_{X^{h}}^{g} \mathcal{Q}\right) Y^{h}\right]=0
$$

This condition is equivalent to

$$
\mathcal{L}_{\xi_{Q}}\left[\mathcal{Q}\left(\nabla_{X^{h}}^{g} Y^{h}\right)\right]=0
$$

But, as $\mathcal{D}$ and $\mathcal{D}^{\perp}$ are $G$-invariant, we have that $\mathcal{L}_{\xi_{Q}} \mathcal{Q}=0$. This, together with (10), imply (11).

Now, we define an affine connection on $M$ as follows: for $X, Y \in \mathfrak{X}(M)$, put

$$
\tilde{\nabla}_{X} Y=\pi_{*}\left(\bar{\nabla}_{X^{h}} Y^{h}\right)
$$


This is well-defined since, by Proposition 4.2, the vector field $\bar{\nabla}_{X^{h}} Y^{h}$ is projectable, and one easily verifies that $\tilde{\nabla}$ satisfies the properties of an affine connection. Then, we obtain the following important result.

Proposition 4.3.([20]) The geodesics of $\bar{\nabla}$, with initial condition in $\mathcal{D}$, project onto the geodesics of $\tilde{\nabla}$.

Proof. Key fact for the proof is that $\mathcal{D}$ is geodesically invariant with respect to $\bar{\nabla}$.

Consequently, we have found that the equations of motion of the given generalized Chaplygin system reduce to the geodesic equations of the induced affine connection $\tilde{\nabla}$ on $M=Q / G$.

Consider the following (0,3)-tensor field on $Q$ :

$$
K_{q}\left(U_{q}, V_{q}, W_{q}\right)=g_{q}\left(\mathbf{h}_{\gamma} U_{q},\left(\Omega^{\gamma}\left(V_{q}, W_{q}\right)\right)_{Q}(q)\right),
$$

where $\mathbf{h}_{\gamma}$ is the horizontal projector and $\Omega^{\gamma}$ is the curvature of the connection $\gamma$. Observe that $K$ is horizontal, i.e. it vanishes if one of its arguments is a vertical vector, and it is skew-symmetric in its last two arguments. Moreover, one can see that

$$
K_{g q}\left(T \Psi_{g}\left(U_{q}\right), T \Psi_{g}\left(V_{q}\right), T \Psi_{g}\left(W_{q}\right)\right)=K_{q}\left(U_{q}, V_{q}, W_{q}\right)
$$

for all $g \in G$ and $q \in Q$. Consequently, $K$ induces a (0,3)-tensor on the base manifold $M$

$$
\tilde{K}_{x}\left(u_{x}, v_{x}, w_{x}\right)=K_{q}\left(U_{q}, V_{q}, W_{q}\right),
$$

where $\pi(q)=x$ and $U_{q}, V_{q}, W_{q}$ are tangent vectors in $q$ projecting onto $u_{x}, v_{x}, w_{x}$, respectively. $K$ (resp. $\tilde{K})$ is called the metric connection tensor on $Q$ (resp. $M)$.

In [20], it was shown that application of the so-called Hamel's approach to mechanics, leads to two additional affine connections on $M$, whose geodesics are also solutions of the reduced nonholonomic problem. These connections are given by

$$
\left(\nabla_{1}^{H}\right)_{X} Y=\nabla_{X}^{\tilde{g}} Y+B(X, Y), \quad\left(\nabla_{2}^{H}\right)_{X} Y=\nabla_{X}^{\tilde{g}} Y+B(Y, X)
$$

where $B$ is the $(1,2)$-tensor field defined by $\beta(B(X, Y))=\tilde{K}(X, Y, \sharp \tilde{g} \beta)$, for any $\beta \in \Lambda^{1}(Q / G), X$, $Y \in \mathfrak{X}(Q / G)$. In general, the tensor which encodes the difference between an affine connection $\nabla$ on a Riemannian manifold and the Levi-Civita connection corresponding to the Riemannian metric, is called the contorsion of $\nabla$. So, $B$ here represents the contorsion of $\nabla_{1}^{H}$.

The following explicit formula for the connection $\tilde{\nabla}$ was then derived in [20] (up to a minor misprint):

$$
\tilde{\nabla}_{X} Y=\nabla_{X}^{\tilde{g}} Y+\frac{1}{2}(B(X, Y)+B(Y, X)-C(X, Y))
$$

where $C$ is the (1,2)-tensor field implicitly defined by $\beta(C(X, Y))=K(\sharp \tilde{g} \beta, X, Y)$, for arbitrary $\beta \in \Lambda^{1}(Q / G), X, Y \in \mathfrak{X}(Q / G)$. As noted in [20], the average of Hamel's connections, i.e. $\nabla^{H / 2}=$ $\frac{1}{2}\left(\nabla_{1}^{H}+\nabla_{2}^{H}\right)$, in general differs from $\tilde{\nabla}$, because of the skew-symmetric term $C(X, Y)$. 
It is interesting to observe that from Proposition 4.1 one can easily deduce

$$
\tilde{\nabla} \tilde{g}=0
$$

that is, $\tilde{\nabla}$ is a metric connection. From Proposition A.3 (see Appendix) and the definition of $B$, it is readily seen that $\nabla_{1}^{H}$ is also a metric connection. In general, however, the connections $\nabla_{2}^{H}$ and $\nabla^{H / 2}$ will not be metric. In fact, it is not hard to prove the following result.

Proposition 4.4. The following properties are equivalent:

(i) $\nabla_{2}^{H}$ is metric;

(ii) $\nabla^{H / 2}$ is metric;

(iii) the tensor field $B$ is skew-symmetric;

(iv) $\nabla^{H / 2}$ is the Levi-Civita connection of $\tilde{g}$.

Later we will see that these properties are also equivalent to the vanishing of the 1-form $\overline{\alpha_{X}}$ and, hence, to the Hamiltonian nature of the reduced system (cf. Corollary 6.2).

The torsion of $\tilde{\nabla}$ is given by $\tilde{T}(X, Y)=\pi_{*} \bar{T}\left(X^{h}, Y^{h}\right)$, with $\bar{T}$ the torsion of $\bar{\nabla}$. Then, we see from the above that the metric connection $\tilde{\nabla}$ is the Levi-Civita connection associated to $\tilde{g}$ iff the torsion of $\bar{\nabla}$ takes values in the vertical tangent bundle to $\pi$ for each pair of vectors in $\mathcal{D}$.

Finally, the following result shows that equality of $\tilde{\nabla}$ and $\nabla^{H / 2}$ is a rather strong condition. Proposition 4.5.

$$
\tilde{\nabla}=\nabla^{H / 2} \Longleftrightarrow \tilde{\nabla}=\nabla^{H / 2}=\nabla^{\tilde{g}}
$$

Proof. If $\tilde{\nabla}=\nabla^{H / 2}$, then $\nabla^{H / 2}$ is metric. By Proposition 4.4, this implies that $\nabla^{H / 2}$ coincides with $\nabla^{\tilde{g}}$. The reverse implication is trivial.

\subsection{Reconstruction}

In view of Proposition 4.3 above, we see that, in the present setting, the reconstruction of the solution curves in $Q$ of the given constrained system, from those of the reduced system on $M$, consists of a horizontal lift operation with respect to the connection $\gamma$. That is, let $\tilde{c}(t)$ be a geodesic of $\tilde{\nabla}$ and choose $c(0) \in Q$ such that $\pi(c(0))=\tilde{c}(0)$. Then, the geodesic starting at $c(0)$, with initial velocity $\dot{\tilde{c}}(0) \in \mathcal{D}_{c(0)}$, is precisely the horizontal lift of $\tilde{c}(t)$ with respect to the principal connection $\gamma$.

\section{Examples}

\subsection{Mobile robot with fixed orientation}

Consider the motion of a mobile robot whose body maintains a fixed orientation with respect to its environment (see 18] for more details). The robot has three wheels, with radius $R$, which turn 
simultaneously about independent axes, and perform a rolling without sliding over a horizontal floor. Let $(x, y) \in \mathbb{R}^{2}$ denote the position of the center of mass of the robot (in a cartesian reference frame, with horizontal $x$-and $y$-axis), $\theta \in \mathbb{S}^{1}$ the steering angle of the wheels and $\psi \in \mathbb{S}^{1}$ the rotation angle of the wheels in their rolling motion over the floor. The configuration space can then be modelled by $Q=\mathbb{S}^{1} \times \mathbb{S}^{1} \times \mathbb{R}^{2}$.

The Lagrangian function $L$ is the kinetic energy function corresponding to the metric

$$
g=m d x \otimes d x+m d y \otimes d y+J d \theta \otimes d \theta+3 J_{w} d \psi \otimes d \psi
$$

where $m$ is the mass of the robot, $J$ its moment of inertia and $J_{w}$ the axial moment of inertia of each wheel. The constraints are induced by the conditions that the wheels roll without sliding, in the direction in which they "point", and that the instantaneous contact points of the wheels with the floor have no velocity component orthogonal to that direction (cf. [18]):

$$
\begin{aligned}
\dot{x} \sin \theta-\dot{y} \cos \theta & =0, \\
\dot{x} \cos \theta+\dot{y} \sin \theta-R \dot{\psi} & =0 .
\end{aligned}
$$

The constraint distribution $\mathcal{D}$ is then spanned by

$$
\left\{\frac{\partial}{\partial \theta}, \frac{\partial}{\partial \psi}+R\left(\cos \theta \frac{\partial}{\partial x}+\sin \theta \frac{\partial}{\partial y}\right)\right\} .
$$

If we consider the abelian action of $G=\mathbb{R}^{2}$ on $Q$ by translations

$$
\begin{aligned}
& \Psi: \quad G \times Q \quad \longrightarrow \quad Q \\
& ((a, b),(\theta, \psi, x, y)) \longmapsto(\theta, \psi, a+x, b+y),
\end{aligned}
$$

we see that the constraint distribution $\mathcal{D}$ can be interpreted as the horizontal subspace of the principal connection $\gamma=(d x-R \cos \theta d \psi) e_{1}+(d y-R \sin \theta d \psi) e_{2}$, where $\left\{e_{1}, e_{2}\right\}$ is the canonical basis of $\mathbb{R}^{2}$ (identified with the Lie algebra of $G$ ).

The metric induced on $M=Q / G$ here becomes

$$
\tilde{g}=J d \theta \otimes d \theta+\left(3 J_{\omega}+m R^{2}\right) d \psi \otimes d \psi .
$$

The reduced Lagrangian $L^{*}$ is the kinetic energy function corresponding to the metric $\tilde{g}$. Moreover, one easily verifies that the gyroscopic 1 -form $\overline{\alpha_{X}}$ identically vanishes and, hence, the symplectic reduction (8) yields

$$
i_{\bar{X}} \omega_{L^{*}}=d E_{L^{*}},
$$

i.e. the reduced system is an unconstrained, purely Lagrangian system.

On the other hand, one can easily check that, in this example, the metric connection tensor $\tilde{K}$ also vanishes. Consequently, with the notations of the previous section, $\tilde{\nabla}=\nabla^{H / 2}$ and by Proposition 4.5 we then have that $\tilde{\nabla}=\nabla^{H / 2}=\nabla^{\tilde{g}}$. 


\subsection{Two-wheeled planar mobile robot}

Consider the motion of a two-wheeled planar mobile robot (or "two-wheeled carriage") which is able to move in the direction in which it points and, in addition, can spin about a vertical axis [18, 20, 27, 33]. Let $P$ be the intersection point of the horizontal symmetry axis of the robot and the horizontal line connecting the centers of the two wheels. The position and orientation of the robot is determined, with respect to a fixed cartesian reference frame (with horizontal $x$ - and $y$-axes) by $(x, y, \theta) \in S E(2)$, where $\theta \in \mathbb{S}^{1}$ is the heading angle and the coordinates $(x, y) \in \mathbb{R}^{2}$ locate the point $P$ (see [20, 33]). Let $\psi_{1}, \psi_{2} \in \mathbb{S}^{1}$ denote the rotation angles of the wheels which are assumed to be controlled independently and roll without slipping on the floor. The configuration space of this system is $Q=\mathbb{S}^{1} \times \mathbb{S}^{1} \times S E(2)$.

The Lagrangian function is the kinetic energy corresponding to the metric

$$
\begin{aligned}
g= & m d x \otimes d x+m d y \otimes d y+m_{0} l \cos \theta(d y \otimes d \theta+d \theta \otimes d y) \\
& -m_{0} l \sin \theta(d x \otimes d \theta+d \theta \otimes d x)+J d \theta \otimes d \theta+J_{2} d \psi_{1} \otimes d \psi_{1}+J_{2} d \psi_{2} \otimes d \psi_{2},
\end{aligned}
$$

where $m=m_{0}+2 m_{1}, m_{0}$ is the mass of the robot without the wheels, $J$ its moment of inertia with respect to the vertical axis, $m_{1}$ the mass of each wheel, $J_{w}$ the axial moments of inertia of the wheels, and $l$ the distance between the center of mass $C$ of the robot and the point $P$.

The constraints, induced by the conditions that there is no lateral sliding of the robot and that the motion of the wheels also consists of a rolling without sliding are:

$$
\begin{aligned}
\dot{x} \sin \theta-\dot{y} \cos \theta & =0, \\
\dot{x} \cos \theta+\dot{y} \sin \theta+c \dot{\theta}+R \dot{\psi}_{1} & =0, \\
\dot{x} \cos \theta+\dot{y} \sin \theta-c \dot{\theta}+R \dot{\psi}_{2} & =0,
\end{aligned}
$$

where $R$ is the radius of the wheels and $2 c$ the lateral length of the robot. The constraint distribution $\mathcal{D}$ is then spanned by

$$
\left\{\frac{\partial}{\partial \psi_{1}}-\frac{R}{2}\left(\cos \theta \frac{\partial}{\partial x}+\sin \theta \frac{\partial}{\partial y}+\frac{1}{c} \frac{\partial}{\partial \theta}\right), \frac{\partial}{\partial \psi_{2}}-\frac{R}{2}\left(\cos \theta \frac{\partial}{\partial x}+\sin \theta \frac{\partial}{\partial y}-\frac{1}{c} \frac{\partial}{\partial \theta}\right)\right\} .
$$

If we consider the action of $G=S E(2)$ on $Q$

$$
\begin{aligned}
& \Psi: \quad G \times Q \quad \longrightarrow Q \\
& \left((a, b, \alpha),\left(\psi_{1}, \psi_{2}, x, y, \theta\right)\right) \longmapsto\left(\psi_{1}, \psi_{2}, a+x \cos \alpha-y \sin \alpha, b+x \sin \alpha+y \cos \alpha, \alpha+\theta\right),
\end{aligned}
$$

we see that the constraint distribution $\mathcal{D}$ can be interpreted as the horizontal subspace of the principal connection

$$
\begin{aligned}
\gamma= & \left(d x+\frac{R}{2} \cos \theta d \psi_{1}+\frac{R}{2} \cos \theta d \psi_{2}+y\left(d \theta+\frac{R}{2 c} d \psi_{1}-\frac{R}{2 c} d \psi_{2}\right)\right) e_{1} \\
& +\left(d y+\frac{R}{2} \sin \theta d \psi_{1}+\frac{R}{2} \sin \theta d \psi_{2}-x\left(d \theta+\frac{R}{2 c} d \psi_{1}-\frac{R}{2 c} d \psi_{2}\right)\right) e_{2} \\
& +\left(d \theta+\frac{R}{2 c} d \psi_{1}-\frac{R}{2 c} d \psi_{2}\right) e_{3},
\end{aligned}
$$


where $\left\{e_{1}, e_{2}, e_{3}\right\}$ is the canonical basis of the Lie algebra of $G$, with associated fundamental vector fields

$$
\left(e_{1}\right)_{Q}=\frac{\partial}{\partial x}, \quad\left(e_{2}\right)_{Q}=\frac{\partial}{\partial y}, \quad\left(e_{3}\right)_{Q}=\frac{\partial}{\partial \theta}-y \frac{\partial}{\partial x}+x \frac{\partial}{\partial y} .
$$

The curvature of $\gamma$ is

$$
\Omega=\frac{R^{2}}{2 c}\left(\sin \theta e_{1}-\cos \theta e_{2}\right) d \psi_{1} \wedge d \psi_{2}
$$

The induced metric on $M=Q / G$ is given by

$$
\begin{aligned}
\tilde{g}= & \left(J_{2}+m \frac{R^{2}}{4}+J \frac{R^{2}}{4 c^{2}}\right) d \psi_{1} \otimes d \psi_{1} \\
& +\left(m \frac{R^{2}}{4}-J \frac{R^{2}}{4 c^{2}}\right)\left(d \psi_{1} \otimes d \psi_{2}+d \psi_{2} \otimes d \psi_{1}\right)+\left(J_{2}+m \frac{R^{2}}{4}+J \frac{R^{2}}{4 c^{2}}\right) d \psi_{2} \otimes d \psi_{2} .
\end{aligned}
$$

The Lagrangian $L^{*}$ is the kinetic energy function induced by $\tilde{g}$. The gyroscopic 1 -form $\overline{\alpha_{X}}$ here becomes

$$
\overline{\alpha_{X}}=\frac{m_{0} l R^{3}}{4 c^{2}}\left(\dot{\psi}_{2}-\dot{\psi}_{1}\right)\left(\dot{\psi}_{1} d \psi_{2}-\dot{\psi}_{2} d \psi_{1}\right) .
$$

Then, the symplectic reduction (8) yields

$$
i_{\bar{X}} \omega_{L^{*}}=d E_{L^{*}}+\overline{\alpha_{X}}
$$

On the other hand, the metric connection tensor $\tilde{K}$ is given by

$$
\tilde{K}=\frac{m_{0} l R^{3}}{4 c^{2}}\left(d \psi_{1} \otimes d \psi_{1} \wedge d \psi_{2}-d \psi_{2} \otimes d \psi_{1} \wedge d \psi_{2}\right)
$$

It is easily seen that, in this case, the tensor field $B$ is not skewsymmetric and so, by Proposition $4.4, \nabla^{H / 2} \neq \nabla^{\tilde{g}}$. In addition, the Christoffel symbols of the metric connection $\tilde{\nabla}$ are

$$
\begin{aligned}
& \Gamma_{\psi_{1} \psi_{1}}^{\psi_{1}}=K_{1}, \quad \Gamma_{\psi_{1} \psi_{1}}^{\psi_{2}}=K_{2}, \\
& \Gamma_{\psi_{1} \psi_{2}}^{\psi_{1}}=-K_{2}, \quad \Gamma_{\psi_{1} \psi_{2}}^{\psi_{2}}=-K_{1}, \\
& \Gamma_{\psi_{2} \psi_{1}}^{\psi_{1}}=-K_{1}, \quad \Gamma_{\psi_{2} \psi_{1}}^{\psi_{2}}=-K_{2}, \\
& \Gamma_{\psi_{2} \psi_{2}}^{\psi_{1}}=K_{2}, \quad \Gamma_{\psi_{2} \psi_{2}}^{\psi_{2}}=K_{1},
\end{aligned}
$$

where

$$
\begin{aligned}
& K_{1}=m_{0} R^{5} l \frac{J_{2}-m c^{2}}{4 c^{2}\left(2 J c^{2}+j_{2} R^{2}\right)\left(2 J+m c^{2}\right)}, \\
& K_{2}=R^{3} l \frac{4 J c^{2}+\left(J_{2}+m c^{2}\right) R^{2} m_{0}}{4 c^{2}\left(2 J c^{2}+j_{2} R^{2}\right)\left(2 J+m c^{2}\right)} .
\end{aligned}
$$

Clearly, the torsion $\tilde{T}$ does not vanish, and so $\tilde{\nabla} \neq \nabla^{\tilde{g}}$. 


\section{Relation between both approaches}

The above examples show us the following intriguing fact. In the case of the mobile robot with fixed orientation, the 1-form $\overline{\alpha_{X}}$ identically vanishes, and thus the reduced problem has no external gyroscopic force in its unconstrained symplectic formulation. Consequently, since there is no potential, the solutions of the reduced system are geodesics of the Levi-Civita connection $\nabla^{\tilde{g}}$. Indeed, we verified that $\tilde{\nabla}=\nabla^{H / 2}=\nabla^{\tilde{g}}$. However, in the case of the two-wheeled mobile robot we obtained $\overline{\alpha_{X}} \neq 0$ and $\tilde{\nabla} \neq \nabla^{\tilde{g}} \neq \nabla^{H / 2}$. Apparently, there exists a relation between the properties of the contorsions of the connections considered in Section 4 and the vanishing (or not) of the gyroscopic 1-form.

Using the definition of $\alpha_{X}$ in (『), one can check that the following relation holds (see [1]): for any $Y \in \mathfrak{X}(\mathcal{D})$,

$$
\alpha_{X}(Y)=\mathbf{v} \Upsilon(Y)\left(\theta_{L}(X)\right)+\theta_{L}(R(X, Y))+\theta_{L}(\mathbf{h} \Upsilon[X, \mathbf{v} \Upsilon(Y)])
$$

where $R$ is the tensor field of type $(1,2)$ on $\mathcal{D}$ given by

$$
R=\frac{1}{2}\left[\mathbf{h}_{\Upsilon}, \mathbf{h}_{\Upsilon}\right]
$$

with [, ] denoting the Nijenhuis bracket of type $(1,1)$ tensor fields. The relation between $R$ and $\Omega^{\Upsilon}$, the curvature tensor of the principal connection $\Upsilon$, is given by $R(U, V)(v)=\left(\Omega^{\Upsilon}\left(U_{v}, V_{v}\right)\right)_{T Q}(v)$ for any $U, V \in \mathfrak{X}(\mathcal{D})$ and $v \in \mathcal{D}$.

In particular, if we take a horizontal vector field $Y \in \mathcal{U}$, we deduce from the above that $\alpha_{X}(Y)=$ $\theta_{L}(R(X, Y))$. Herewith, the action of the gyroscopic 1 -form $\overline{\alpha_{X}}$ on a vector field $Z \in \mathfrak{X}(T(Q / G))$, evaluated at a point $w_{\bar{q}} \in T(Q / G)(\cong \overline{\mathcal{D}})$, becomes

$$
\begin{aligned}
\overline{\alpha_{X}}(Z)\left(w_{\bar{q}}\right) & =\alpha_{X}\left(Z^{h}\right)\left(v_{q}\right)=\left(\theta_{L}\right)_{v_{q}}\left(R\left(X, Z^{h}\right)\left(v_{q}\right)\right) \\
& =\left(\theta_{L}\right)_{v_{q}}\left(\left(\Omega^{\Upsilon}\left(X_{v_{q}}, Z_{v_{q}}^{h}\right)\right)_{T Q}\left(v_{q}\right)\right)=\left(\theta_{L}\right)_{v_{q}}\left(\left(\Omega^{\gamma}\left(\tau_{Q_{*}} X_{v_{q}}, \tau_{Q_{*}} Z_{v_{q}}^{h}\right)\right)_{T Q}\left(v_{q}\right)\right),
\end{aligned}
$$

for an arbitrary $v_{q} \in \mathcal{D}$ such that $\rho\left(v_{q}\right)=w_{\bar{q}}$, and where the last equality has been derived using (6)). In these expressions, $Z^{h}$ is the horizontal lift of $Z$ with respect to $\Upsilon$. Recalling that the PoincaréCartan 1-form $\theta_{L}$ and the Legendre mapping (induced by the given Lagrangian) $F L: T Q \longrightarrow T^{*} Q$ are related by $\left(\theta_{L}\right)_{v_{q}}(u)=<F L\left(v_{q}\right), \tau_{Q_{*}}(u)>$, for any $u \in T_{v_{q}} T Q$, and taking into account that $X$ is a SODE, we further obtain

$$
\begin{aligned}
\overline{\alpha_{X}}(Z)\left(w_{\bar{q}}\right) & =<F L\left(v_{q}\right),\left(\Omega^{\gamma}\left(\tau_{Q_{*}} X_{v_{q}}, \tau_{Q_{*}} Z_{v_{q}}^{h}\right)\right)_{Q}(q)> \\
& =g_{q}\left(v_{q},\left(\Omega^{\gamma}\left(v_{q}, \tau_{Q_{*}} Z_{v_{q}}^{h}\right)\right)_{Q}(q)\right) \\
& =g_{q}\left(v_{q},\left(\Omega^{\gamma}\left(v_{q},\left(\tau_{Q / G_{*}} Z_{w_{\bar{q}}}\right)_{q}^{h}\right)\right)_{Q}(q)\right) .
\end{aligned}
$$

Note that in the last expression, the horizontal lift of $\tau_{Q / G_{*}} Z_{w_{\bar{q}}}$ is the one with respect to $\gamma$.

An important observation is that (13) immediately shows that the gyroscopic 1-form $\overline{\alpha_{X}}$ is semibasic with respect to the canonical fibration $\tau_{Q / G}: T(Q / G) \longrightarrow Q / G$. Indeed, assume $\left(\tau_{Q / G}\right)_{*} \circ Z=$ 0 , then it readily follows that $\overline{\alpha_{X}}(Z)=0$. 
Elaborating (13) a bit further, using the metric connection tensor $\tilde{K}$ and the contorsion $B$ introduced in Section 4.1, we find

$$
\begin{aligned}
\overline{\alpha_{X}}(Z)\left(w_{\bar{q}}\right) & \left.=g_{q}\left(v_{q}, \Omega\left(v_{q},\left(\tau_{Q / G_{*}} Z_{w_{\bar{q}}}\right)_{q}^{h}\right)\right)_{Q}(q)\right) \\
& =\tilde{K}_{\bar{q}}\left(w_{\bar{q}}, w_{\bar{q}}, \tau_{Q / G_{*}} Z_{w_{\bar{q}}}\right) \\
& =\tilde{g}_{\bar{q}}\left(B\left(w_{\bar{q}}, w_{\bar{q}}\right), \tau_{Q / G_{*}} Z_{w_{\bar{q}}}\right)
\end{aligned}
$$

This proves the next result, which was already implicit in the work of Koiller [20].

Proposition 6.1. An explicit relation between the gyroscopic 1-form and the contorsion tensor field $B$, defined in Section 4 , is given by

$$
\left(\overline{\alpha_{X}}\right)_{w_{\bar{q}}}(u)=\tilde{g}_{\bar{q}}\left(B\left(w_{\bar{q}}, w_{\bar{q}}\right), \tau_{Q / G_{*}} u\right),
$$

for all $\left.u \in T_{w_{\bar{q}}} T(Q / G)\right), w_{\bar{q}} \in T(Q / G)$.

From this we can immediately deduce, taking into account Proposition 4.4:

Corollary 6.2. The following statements are equivalent:

(i) $\overline{\alpha_{X}}$ vanishes identically;

(ii) B is skew-symmetric;

(iii) $\nabla^{H / 2}=\nabla^{\tilde{g}}$.

REMARK 6.3. One can think of simple examples in which $B$ is skew-symmetric but nonzero. Consequently, if $\overline{\alpha_{X}}$ vanishes, this does not imply $\tilde{\nabla}=\nabla^{\tilde{g}}$, although in such a case both connections do have the same geodesics (see Appendix).

By means of Proposition 6.1, one can also recover the gyroscopic character of $\overline{\alpha_{X}}$, established already in Proposition 3.4. For that purpose, let us define the following 2-form on $T(Q / G)$ :

$$
\Xi(Y, Z)\left(w_{\bar{q}}\right)=\tilde{g}_{\bar{q}}\left(B\left(w_{\bar{q}}, \tau_{Q / G_{*}} Y_{w_{\bar{q}}}\right), \tau_{Q / G_{*}} Z_{w_{\bar{q}}}\right) .
$$

One readily verifies that $\Xi$ is indeed bilinear and by Proposition A.3, $\Xi(Y, Y)=0$. It is then easy to check that

$$
\overline{\alpha_{X}}=i_{\bar{X}} \Xi
$$

In local coordinates $q^{a}(a=1, \ldots, n-k)$ on $M=Q / G$, we have that

$$
\Xi=\sum_{a<b} \dot{q}^{e} B_{e a}^{c} \tilde{g}_{b c} d q^{a} \wedge d q^{b}, \quad \overline{\alpha_{X}}=\sum_{a, e, c} \dot{q}^{a} \dot{q}^{e} B_{e a}^{c} \tilde{g}_{b c} d q^{b}
$$

A careful calculation, very similar to the one performed for proving Proposition 6.1, reveals that for generalized Chaplygin systems of mechanical type, the 2-form $\bar{\Sigma}$ of Proposition 3.4 and the above 2-form $\Xi$ coincide. 


\section{Integrability aspects and the existence of an invariant measure}

\subsection{Koiller's question}

In [20], the author wonders whether there might exist an invariant measure for the reduced equations of a generalized Chaplygin system. In the following, we shall deal with this problem in some detail.

The existence of a measure which is invariant under the flow of a given dynamical system is a strong property. Indeed, using an integrating factor it is possible to derive from it (locally) an integral of the motion. This fact plays an important role in discussions concerning the integrability of the system under consideration, as illustrated by the following theorem.

TheOREm 7.1.(运) Suppose that the system $\dot{x}=X(x), x \in N$, with $N$ an $n$-dimensional smooth manifold, admits an invariant measure and has $n-2$ first integrals $F_{1}, \ldots, F_{n-2}$. Suppose also that $F_{1}, \ldots, F_{n-2}$ are independent on the invariant set $N_{c}=\left\{x \in N: F_{s}(x)=c_{s}, 1 \leq s \leq n-2\right\}$. Then:

- the solutions of the differential equation lying on $N_{c}$ can be found by quadratures.

Moreover, if $L_{c}$ is a compact connected component of the level set $N_{c}$ and if $X$ does not vanish on $L_{c}$, then

- $L_{c}$ is a smooth manifold diffeomorphic to a two-torus;

- one can find angular coordinates $\varphi_{1}, \varphi_{2} \bmod (2 \pi)$ on $L_{c}$ in terms of which the differential equations take the simple form

$$
\dot{\varphi}_{1}=\frac{\omega_{1}}{\Phi\left(\varphi_{1}, \varphi_{2}\right)}, \quad \dot{\varphi}_{2}=\frac{\omega_{2}}{\Phi\left(\varphi_{1}, \varphi_{2}\right)},
$$

where $\omega_{1}, \omega_{2}$ are constant and $\Phi$ is a smooth positive function which is $2 \pi$-periodic in $\varphi_{1}$, $\varphi_{2}$.

By the Riesz representation theorem, we know that each volume form on an orientable manifold induces a unique measure on the Borel $\sigma$-algebra [1]. Therefore, with a view on tackling the integrability problem of generalized Chaplygin systems, it is worth looking for invariant volume forms under the flow of the reduced dynamics $\bar{X}$. This is what we intend to do in the sequel.

In this section, we again consider Chaplygin systems of mechanical type with a Lagrangian $L=$ $\frac{1}{2} g-V$, where the metric $g$ and the potential energy function $V$ are $G$-invariant. The reduced equations of motion are (cf. (8))

$$
i_{\bar{X}} \omega_{L^{*}}=d E_{L^{*}}+\overline{\alpha_{X}},
$$

where $L^{*}=\frac{1}{2} \tilde{g}-\tilde{V}$, with $\tilde{g}$ and $\tilde{V}$ the metric and the potential function on $M$ induced by, respectively, $g$ and $V$. Remember that the energy $E_{L^{*}}$ is a constant of the motion. The local expression for the reduced dynamics takes the form (cf. (9))

$$
\bar{X}=\dot{q}^{a} \frac{\partial}{\partial q^{a}}-\left(\tilde{g}^{a b}\left(\alpha_{b}+\frac{\partial \tilde{V}}{\partial q^{b}}\right)+\dot{q}^{b} \dot{q}^{c} \tilde{\Gamma}_{b c}^{a}\right) \frac{\partial}{\partial \dot{q}^{a}},
$$


where $\tilde{\Gamma}_{b c}^{a}$ are the Christoffel symbols of the Levi-Civita connection $\nabla^{\tilde{g}}$.

The gyroscopic systems usually encountered in the mechanics literature 42, 43, differ in a crucial way from the ones we obtain through the reduction of a generalized Chaplygin system. In fact, the common situation in mechanics is that of a system, with configuration space $P$, described by an equation of the form

$$
i_{\Gamma} \omega_{\mathbb{L}}=d E_{\mathbb{L}}+\alpha
$$

where $\mathbb{L}: T P \longrightarrow \mathbb{R}$ is a (regular) Lagrangian and where the gyroscopic force is represented by a 1form $\alpha=i_{\Gamma}\left(\tau_{P}^{*} \Pi\right)$, with $\Pi$ a closed 2-form on $P$. These systems are then Hamiltonian with respect to the symplectic 2 -form $\omega=\omega_{\mathbb{L}}-\tau_{P}^{*} \Pi$, and thus they admit an invariant measure, determined by the volume form $\omega^{n}=\omega_{\mathbb{L}}^{n}$.

In some sense, our reduced system (16) exhibits the opposite behaviour. Indeed, the 2 -form $\Xi$, defined by (14), is semi-basic but in general it is not basic, i.e. it is not the pull-back of a 2-form on the base $M=Q / G$. This can be readily deduced from its local expression (cf. 15). Moreover, using (15), the following property is easily proved.

Proposition 7.2. The 2-form $\Xi$ is closed if and only if it is identically zero.

Note, in passing, that a similar property also applies to the gyroscopic 1-form $\overline{\alpha_{X}}$. The semi-basic character of $\Xi$ ensures, however, that the 2-form $\omega_{L^{*}}-\Xi$ is still nondegenerate and, consequently, we have that the equation (16) can be rewritten in the form

$$
i_{\bar{X}} \omega=d E_{L^{*}}
$$

with $\omega=\omega_{L^{*}}-\Xi$ an almost symplectic form (i.e. a nondegenerate, but not necessarily closed 2 -form).

In [36], S.V. Stanchenko has studied Chaplygin systems of mechanical type with an abelian Lie group in terms of differential forms, in a way which shows many links to the approach described in Section 3. In our setting, his results can be generalized to the non-abelian case for any kind of generalized Chaplygin system.

Let us assume, following [36], that there exists a function $F \in C^{\infty}(T(Q / G))$ such that

$$
d F \wedge \theta_{L^{*}}=\Xi
$$

Putting $N=\exp F$, we have that

$$
d(N \omega)=d\left(N \omega_{L^{*}}-N \Xi\right)=d\left(N \omega_{L^{*}}-d N \wedge \theta_{L^{*}}\right)=0 .
$$

Since $(17)$ can still be written as

$$
i_{\bar{X} / N}(N \omega)=d E_{L^{*}},
$$

we deduce that $\mathcal{L}_{\bar{X} / N}(N \omega)=0$. Consequently,

$$
0=\mathcal{L}_{\bar{X} / N}(N \omega)^{n}=\mathcal{L}_{\bar{X}} N^{n-1} \omega^{n}
$$


and we see that $N^{n-1} \omega^{n}$ is an invariant volume form. This proves the following result.

Theorem 7.3.([36]) Condition (18) is sufficient for the existence of an invariant measure for the reduced Chaplygin equations (17).

REMARK 7.4. Stanchenko observes that if $F$ satisfies (18), the semi-basic character of both $\theta_{L^{*}}$ and $\Xi$ imply that $F$ is necessarily the pullback of a function on $Q / G(=M)$.

It turns out that condition (18) can be relaxed to some extent: it suffices to require the almost symplectic 2-form $\omega$ to be globally conformal symplectic, that is, that there exists a function $F \in C^{\infty}(T(Q / G))$ such that

$$
d F \wedge \omega=-d \omega .
$$

Theorem 7.3 still holds in this case, with (19) replacing (18). The previous remark also remains valid: the function $F$ is necessarily the pullback of a function on $Q / G$. Note that (18) obviously implies (19).

However, even the weaker condition (19) is not necessary in general for the existence of an invariant volume form on $T(Q / G)$. To derive a necessary condition, let us suppose that $\mu$ is an invariant volume form for the dynamics $\bar{X}$ on $T(Q / G)$. We then necessarily have that $\mu=k \omega^{n}$, for some nowhere vanishing function $k \in C^{\infty}(T(Q / G))$. Restricting ourselves to a connected component of $Q / G$ if need be, we may always assume $k$ is strictly positive. It follows that

$$
\begin{aligned}
0=\mathcal{L}_{\bar{X}} \mu & =\bar{X}(k) \omega^{n}+k \mathcal{L}_{\bar{X}} \omega^{n} \\
& =\bar{X}(k) \omega^{n}+n k \mathcal{L}_{\bar{X}} \omega \wedge \omega^{n-1}=\bar{X}(k) \omega^{n}-n k i_{\bar{X}} d \Xi \wedge \omega^{n-1}
\end{aligned}
$$

The $2 n$-form $i_{\bar{X}} d \Xi \wedge \omega^{n-1}$ determines a function $h \in C^{\infty}(T(Q / G))$ by

$$
i_{\bar{X}} d \Xi \wedge \omega^{n-1}=\frac{h}{n} \omega^{n}
$$

Therefore, we have

$$
\bar{X}(k)=k h \quad \text { or, equivalently, } \quad \bar{X}(\ln k)=h .
$$

This essentially yields the same characterization as the one derived in [36]. Now, conversely, assume there exists a function $k$ satisfying (21), with $h$ defined by (20). Going through the above computations in reverse order then shows that the $2 n$-form $k \omega^{n}$ is an invariant volume form of $\bar{X}$. We may therefore conclude that the existence of a globally defined function $k$ for which (21) holds is not only a necessary but also a sufficient condition for the existence of an invariant volume form. It is interesting to note that in 36], Stanchenko has proved that in case the reduced Lagrangian is of kinetic energy type, $L^{*}=\frac{1}{2} \tilde{g}$, and if there exists a solution $k$ of (21) which is basic, i.e. which is the pullback of a function on the base space $Q / G$, then the volume form $\mu=k \omega^{n}$ remains an invariant of the reduced dynamics if a potential energy function $\tilde{V} \in C^{\infty}(Q / G)$ is included in the Lagrangian $L^{*}$ (coming from a $G$-invariant potential added to the given Chaplygin system).

Obviously, however, (21) is not a very handy criterium to deal with in practice. We will now see that, at least for a subclass of generalized Chaplygin systems, it may be replaced by a more easily manegeable condition. 
From (20), we can deduce a local expression for $h$. After some computations, we get

$$
h=\sum_{a, b} \tilde{g}^{a b} \frac{\partial \alpha_{b}}{\partial \dot{q}^{a}},
$$

and, using (15), this further becomes

$$
h=\sum_{f, b} \tilde{g}^{f b} \dot{q}^{e} \tilde{g}_{b c}\left(B_{e f}^{c}+B_{f e}^{c}\right)=\sum_{c, e} \dot{q}^{e}\left(B_{e c}^{c}+B_{c e}^{c}\right) .
$$

Note that $S^{*} d h$ is the pullback of a basic 1-form, i.e. $S^{*} d h=\tau_{Q / G}^{*} \beta$, where the local expression for $\beta$ reads

$$
\beta=h_{e}(q) d q^{e}=\sum_{c}\left(B_{e c}^{c}+B_{c e}^{c}\right) d q^{e},
$$

with $h_{e}=\partial h / \partial \dot{q}^{e}$. Let us assume now that there exists a basic function $k$ for which (21) holds. We then have that $S^{*}(d \bar{X}(\ln k))=d(\ln k)$. Therefore, taking the differential of both hand-sides of (21) and applying $S^{*}$ to the resulting equality, we obtain

$$
d(\ln k)=\beta,
$$

or, in local coordinates,

$$
\frac{\partial(\ln k)}{\partial q^{e}}=h_{e}(q)=\sum_{c}\left(B_{e c}^{c}+B_{c e}^{c}\right), e=1, \ldots, n .
$$

We thus see that, if (21) admits a solution $k$ which is basic, then the 1 -form $\beta$ is exact.

It turns out that, for systems for which $\tilde{V}=0$ (i.e. $L^{*}$ is a pure kinetic energy Lagrangian), the previous result even has a more definitive character. Indeed, let $\tilde{V}=0$ and suppose there exists a function $k \in C^{\infty}(T(Q / G)$ ) (not necessarily basic) satisfying (21). Then, we have that

$$
S^{*} d \bar{X}(\ln k)=S^{*} d h
$$

In local coordinates, this becomes

$$
\frac{\partial \bar{X}(\ln k)}{\partial \dot{q}^{e}}=h_{e}(q), e=1, \ldots, n .
$$

But $\bar{X}(\ln k)=\dot{q}^{a} \frac{\partial(\ln k)}{\partial q^{a}}+\bar{X}^{a} \frac{\partial(\ln k)}{\partial \dot{q}^{a}}$, where $\bar{X}^{a}=-\left(\tilde{g}^{a b} \alpha_{b}+\dot{q}^{b} \dot{q}^{c} \tilde{\Gamma}_{b c}^{a}\right)$, and so we have

$$
\frac{\partial \bar{X}(\ln k)}{\partial \dot{q}^{e}}=\frac{\partial(\ln k)}{\partial q^{e}}+\dot{q}^{a} \frac{\partial^{2}(\ln k)}{\partial q^{a} \partial \dot{q}^{e}}+\frac{\partial \bar{X}^{a}}{\partial \dot{q}^{e}} \frac{\partial(\ln k)}{\partial \dot{q}^{a}}+\bar{X}^{a} \frac{\partial^{2}(\ln k)}{\partial \dot{q}^{a} \partial \dot{q}^{e}} .
$$

In points $0_{q}$ of the zero section of $T(Q / G)$ this reduces to

$$
\left.\frac{\partial \bar{X}(\ln k)}{\partial \dot{q}^{e}}\right|_{0_{q}}=\left.\frac{\partial(\ln k)}{\partial q^{e}}\right|_{0_{q}} .
$$


If we now define the basic function $\tilde{k}=k \circ s$, where $s: Q / G \longrightarrow T(Q / G), q \longmapsto(q, 0)$ determines the zero section, we derive from the above that

$$
\frac{\partial(\ln \tilde{k})}{\partial q^{e}}(q)=\frac{\partial(\ln k)}{\partial q^{e}}(q, 0)=h_{e}(q), e=1, \ldots, n,
$$

and, hence, it follows again that the 1 -form $\beta$ is exact.

Conversely, if the 1-form $\beta$ is exact, say $\beta=d f$ for some function $f \in C^{\infty}(Q / G)$, and putting $k=\exp \left(\tau_{Q} / G^{*} f\right)$, one easily verifies that $k$ satisfies (21). This obviously also holds in the presence of a potential (i.e. if $\tilde{V} \neq 0$ ).

Summarizing the above, we have proved the following interesting result.

THEOREM 7.5. For a generalized Chaplygin system with a Lagrangian of kinetic energy type, i.e. $L=\frac{1}{2} g$, there exists an invariant volume form for the reduced dynamics $\bar{X}$ on $T(Q / G)$ iff the basic 1-form $\beta$, defined by $S^{*} d h=\tau_{Q / G}^{*} \beta$ (with $h$ given by (20)), is exact. The 'if' part also holds if $L$ is of the form $L=\frac{1}{2} g-V$, with $V$ a $G$-invariant potential.

Therefore, if we manage to find a particular example of a system with a kinetic energy type Lagrangian for which $\beta$ is not exact, we shall have proved that the answer to Koiller's question about the existence of an invariant volume form for all generalized Chaplygin systems, is negative. In particular, for such a counter example it suffices to show that the corresponding $\beta$ is not closed.

\subsection{A counter example}

Let us consider the following modified version of the classical example of the nonholonomic free particle [5].

Consider a particle moving in space, so $Q=\mathbb{R}^{3}$, subject to the non-holonomic constraint

$$
\phi=\dot{z}-y x \dot{x} .
$$

The Lagrangian function is the kinetic energy corresponding to the standard metric $g=d x \otimes d x+$ $d y \otimes d y+d z \otimes d z$. Therefore,

$$
L=\frac{1}{2}\left(\dot{x}^{2}+\dot{y}^{2}+\dot{z}^{2}\right)
$$

The constraint submanifold is defined by the distribution

$$
\mathcal{D}=<\frac{\partial}{\partial x}+y x \frac{\partial}{\partial z}, \frac{\partial}{\partial y}>
$$

Consider the Lie group $G=\mathbb{R}$ with its trivial action by translations on $Q$ :

$$
\begin{aligned}
\Phi: \quad G \times Q & \longrightarrow Q \\
(s,(x, y, z)) & \longmapsto(x, y, z+s) .
\end{aligned}
$$

Note that $\mathcal{D}$ is the horizontal subspace of a connection $\gamma$ on the principal fiber bundle $Q \longrightarrow Q / G$, where $\gamma=d z-y x d x$. Therefore, this system belongs to the class of generalized Chaplygin systems. 
The curvature of $\gamma$ is given by

$$
\Omega^{\gamma}=x d x \wedge d y \text {. }
$$

The induced metric $\tilde{g}$ on $Q / G \cong \mathbb{R}^{2}$ is

$$
\tilde{g}=\left(1+x^{2} y^{2}\right) d x \otimes d x+d y \otimes d y .
$$

The metric connection tensor $\tilde{K}$ is determined by

$$
\tilde{K}\left(\frac{\partial}{\partial x}, \frac{\partial}{\partial x}, \frac{\partial}{\partial y}\right)=x^{2} y, \tilde{K}\left(\frac{\partial}{\partial y}, \frac{\partial}{\partial x}, \frac{\partial}{\partial y}\right)=0 .
$$

Then, the contorsion of the affine connection $\nabla_{1}^{H}$ here reads

$$
B=x^{2} y d x \otimes d x \otimes \frac{\partial}{\partial y}-\frac{x^{2} y}{1+x^{2} y^{2}} d x \otimes d y \otimes \frac{\partial}{\partial x} .
$$

Finally, the 1-form $\beta$ associated to the reduced Chaplygin system is given by

$$
\beta=-\frac{x^{2} y}{1+x^{2} y^{2}} d y
$$

which is clearly not closed. Hence, according to Theorem 7.5, there is no invariant volume form for the reduced dynamics.

Note that in this example the distribution $\mathcal{D}$ has 'length' 1 at all points of $\mathbb{R}^{3}$ not belonging to the plane $x=0$, since $\mathcal{D}+[\mathcal{D}, \mathcal{D}]$ spans the full tangent space at all points for which $x \neq 0$. We leave it as a challenge for the reader to find a more generic example (on $\mathbb{R}^{3}$ ) for which the constraint distribution has length 1 everywhere.

REMARK 7.6. The classical model for the nonholonomic free particle corresponds to the constraint $\phi=\dot{z}-y \dot{x}$. Here the constraint distribution does have length 1 everywhere, however, after performing the appropriate computations, we obtain $\beta=-\frac{y}{1+y^{2}} d y$, which is clearly exact: $\beta=$ $d f$, with $f=\frac{1}{2} \ln \left(\frac{1}{1+y^{2}}\right)$. Then, $k=\frac{1}{\sqrt{1+y^{2}}}$ and the invariant measure defined by $k \omega^{n}$ leads, using Euler's integrating factor technique, to the constant of the motion

$$
\varphi=\dot{x} \sqrt{1+y^{2}}
$$

which was also obtained by different methods in 4, , 1, 13].

\section{Appendix: Metric connections}

In this section, we want to collect some simple facts about metric connections that have been useful for the formulation of generalized Chaplygin systems.

Let $Q$ be a $n$-dimensional differentiable manifold, the configuration space with Riemannian metric $g$. We denote by $\nabla^{g}$ the Levi-Civita or Riemannian connection associated to the metric $g$. 
Definition A.1. An affine connection $\nabla$ is called metric with respect to $g$ if $\nabla g=0$, that is,

$$
Z(g(X, Y))=g\left(\nabla_{Z} X, Y\right)+g\left(X, \nabla_{Z} Y\right)
$$

for all $X, Y, Z \in \mathfrak{X}(Q)$.

Let $\nabla$ be a metric connection with respect to $g$. In the following proposition, we prove that $\nabla$ is determined by its torsion $T$.

Proposition A.2. Let $T$ be a skewsymmetric (1,2) tensor on $Q$. Then there exists a unique metric connection $\nabla$ whose torsion is precisely $T$.

Proof. Let us suppose that there exists such metric connection $\nabla$. Then we have that

$$
\begin{aligned}
& Z(g(X, Y))=g\left(\nabla_{Z} X, Y\right)+g\left(X, \nabla_{Z} Y\right), \\
& X(g(Z, Y))=g\left(\nabla_{X} Z, Y\right)+g\left(Z, \nabla_{X} Y\right), \\
& Y(g(X, Z))=g\left(\nabla_{Y} X, Z\right)+g\left(X, \nabla_{Y} Z\right),
\end{aligned}
$$

for all $X, Y, Z \in \mathfrak{X}(Q)$. Now

$$
\begin{aligned}
& Z(g(X, Y))+X(g(Z, Y))-Y(g(X, Z)) \\
= & g\left(\nabla_{X} Z+\nabla_{Z} X, Y\right)+g\left(\nabla_{Z} Y-\nabla_{Y} Z, X\right)+g\left(\nabla_{X} Y-\nabla_{Y} X, Z\right) \\
= & g\left(2 \nabla_{X} Z+T(Z, X)+[Z, X], Y\right)+g(T(Z, Y)+[Z, Y], X)+g(T(X, Y)+[X, Y], Z) \\
= & 2 g\left(\nabla_{X} Z, Y\right)+g(T(Z, X)+[Z, X], Y)+g(T(Z, Y)+[Z, Y], X)+g(T(X, Y)+[X, Y], Z) .
\end{aligned}
$$

Consequently, the connection $\nabla$ is uniquely determined by the formula

$$
g\left(\nabla_{X} Z, Y\right)=g\left(\nabla_{X}^{g} Z, Y\right)-\frac{1}{2}(g(Y, T(X, Z))+g(X, T(Z, Y))+g(Z, T(X, Y))) .
$$

This proposition implies that the Christoffel symbols $\bar{\Gamma}_{B C}^{A}$ of the metric connection $\nabla$ in a local chart $\left(q^{A}\right)$ are given by

$$
\bar{\Gamma}_{B C}^{A}=\Gamma_{B C}^{A}-\frac{1}{2} g^{A K}\left(g_{K M} T_{B C}^{M}+g_{B M} T_{C K}^{M}+g_{C M} T_{B K}^{M}\right)
$$

where $\Gamma_{B C}^{A}$ are the Christoffel symbols of the connection $\nabla^{g}$ and $T=T_{A B}^{C} d q^{A} \otimes d q^{B} \otimes \frac{\partial}{\partial q^{C}}$.

Another way to characterize metric connections is the following. Given any affine connection on $Q$, we know that

$$
\nabla_{X} Y=\nabla_{X}^{g} Y+S(X, Y)
$$

where $S$ is a $(1,2)$ tensor field, called the contorsion. If $\nabla$ is a metric connection, then

$$
\begin{aligned}
Z(g(X, Y)) & =g\left(\nabla_{Z} X, Y\right)+g\left(X, \nabla_{Z} Y\right) \\
& =g\left(\nabla_{Z}^{g} X+S(Z, X), Y\right)+g\left(X, \nabla_{Z}^{g} Y+S(Z, Y)\right) \\
& =Z(g(X, Y))+g(S(Z, X), Y)+g(X, S(Z, Y)),
\end{aligned}
$$


which implies that $g(S(Z, X), Y)+g(X, S(Z, Y))=0$. Then we have proved the following Proposition A.3. $\nabla$ is a metric connection if and only if

$$
g(S(Z, X), X)=0 \quad \forall X, Z \in \mathfrak{X}(Q)
$$

As a consequence of the two characterizations we have obtained for metric connections, we can establish the next result.

Corollary A.4. There is a one-to-one correspondence between (1,2) tensors $S$ verifying (2) and skewsymmetric (1,2) tensors $T$. This correspondence is given by

$$
S \longrightarrow T
$$

where $T(X, Y)=S(X, Y)-S(Y, X)$ and

$$
T \longrightarrow S
$$

where $g(S(X, Z), Y)=-\frac{1}{2}((g(Y, T(X, Z))+g(X, T(Y, Z))+g(Z, T(X, Y)))$.

The equations for the geodesics of a metric connection are

$$
\nabla_{\dot{c}(t)} \dot{c}(t)=0 \Longleftrightarrow \nabla_{\dot{c}(t)}^{g} \dot{c}(t)=-S(\dot{c}(t), \dot{c}(t))
$$

or, in local coordinates

$$
\ddot{q}^{A}+\Gamma_{B C}^{A} \dot{q}^{B} \dot{q}^{C}=\sum_{B<C} g^{A K}\left(g_{B M} T_{C K}^{M}+g_{C M} T_{B K}^{M}\right) \dot{q}^{B} \dot{q}^{C}
$$

for each $A=1, \ldots, n$.

Finally, it is very important to note that the metric connections preserve obviously the kinetic energy $K$ of the metric $g$, that is, if $c(t)$ is a geodesic of $\nabla$, we have that

$$
\frac{d}{d t}\left(\frac{1}{2} g(\dot{c}(t), \dot{c}(t))=g\left(\nabla_{\dot{c}(t)} \dot{c}(t), \dot{c}(t)\right)=0 .\right.
$$

ACKnowledgements. This work was partially supported by grant DGICYT (Spain) PB97-1257. J. Cortés wishes to thank the Spanish Ministerio de Educación y Cultura for a FPU grant and both J. Cortés and D. Martín de Diego wish to thank the Department of Mathematical Physics and Astronomy of the University of Ghent for its kind hospitality. We would like to thank S. Martínez for helpful conversations and the referee for several useful comments.

\section{References}

[1] R. Abraham and J.E. Marsden. Foundations of Mechanics (2nd Edition) (BenjaminCummings, Reading (Ma), 1978). 
[2] V.I. Arnold. Dynamical Systems III (Springer-Verlag, Berlin, 1988).

[3] L. Bates and R. Cushman. What is a completely integrable nonholonomic dynamical system? Rep. Math. Phys. 44 (1999), 29-35.

[4] L. Bates, H. Graumann and C. MacDonnell. Examples of gauge conservation laws in nonholonomic systems. Rep. Math. Phys. 37 (1996), 295-308.

[5] L. Bates and J. Śniatycki. Nonholonomic reduction. Rep. Math. Phys. 32 (1992), 99-115.

[6] A.M. Bloch and P.E. Crouch. Newton's law and integrability of nonholonomic systems. SIAM J. Control Optim. 36 (1998), 2020-2039.

[7] A.M. Bloch, P.S. Krishnaprasad, J.E. Marsden and R.M. Murray. Nonholonomic mechanical systems with symmetry. Arch. Rational Mech. Anal. 136 (1996), 21-99.

[8] F. Bullo. Series expansions for the evolution of mechanical control systems. Preprint (1999) (http://motion.csl.uiuc.edu/〜bullo/papers).

[9] F. Bullo, N.E. Leonard and A.D. Lewis. Controllability and motion algorithms for underactuated Lagrangian systems on Lie groups. IEEE Trans. Automat. Control 45 (2000), $1437-1454$.

[10] F. Cantrijn, M. de León, J.C. Marrero and D. Martín de Diego. Reduction of nonholonomic mechanical systems with symmetries. Rep. Math. Phys. 42 (1998), 25-45.

[11] F. Cantrijn, M. de León, J.C. Marrero and D. Martín de Diego. Reduction of constrained systems with symmetries. J. Math. Phys. 40 (1999), 795-820.

[12] F. Cantrijn, M. de León and D. Martín de Diego. On almost-Poisson structures in nonholonomic mechanics. Nonlinearity 12 (1999), 721-737.

[13] J. CortÉs and M. DE LEÓN. Reduction and reconstruction of the dynamics of nonholonomic systems. J. Phys. A: Math. Gen. 32 (1999), 8615-8645.

[14] J. Cortés and S. Martínez. Characterization of local controllability for a class of mechanical systems. Preprint (2000) (math.OC/0009095).

[15] R. Cushman, D. Kemppainen, J. Śniatycki and L. Bates. Geometry of nonholonomic constraints. Rep. Math. Phys. 36 (1995), 275-286.

[16] V. Dragović, B. Gajić and B. Jovanović. Generalizations of classical integrable nonholonomic rigid body systems. J. Phys. A: Math. Gen. 31 (1998), 9861-9869.

[17] B. Jovanović. Nonholonomic geodesic flows on Lie groups and the integrable Suslov problem on SO(4). J. Phys. A: Math. Gen. 31 (1998), 1415-1422. 
[18] S.D. Kelly and R.M. Murray. Geometric phases and robotic locomotion. J. Robotic Systems 12 (1995), 417-431.

[19] S. Kobayashi and K. Nomizu. Foundations of Differential Geometry. Interscience Tracts in Pure and Applied Mathematics (Interscience Publishers, Wiley, New-York, 1963).

[20] J. Kolller. Reduction of some classical non-holonomic systems with symmetry. Arch. Rational Mech. Anal. 118 (1992), 113-148.

[21] W.S. Koon and J.E. Marsden. The Hamiltonian and Lagrangian approaches to the dynamics of nonholonomic systems. Rep. Math. Phys. 40 (1997), 21-62.

[22] W.S. Koon and J.E. Marsden. Poisson reduction of nonholonomic mechanical systems with symmetry. Rep. Math. Phys. 42 (1998), 101-134.

[23] V.V. KozLov. Invariant measures of the Euler-Poincaré equations on Lie algebras. Funkt. Anal. Prilozh. 22 (1988), 69-70 (in Russian), Funct. Anal. Appl. 22 (1988) (Engl. transl.)

[24] O. Krupkova. Mechanical systems with nonholonomic constraints. J. Math. Phys. 38 (1997), $5098-5126$.

[25] M. De León and P.R. Rodrigues. Methods of Differential Geometry in Analytical Mechanics. Math. Ser. 152 (North-Holland, Amsterdam, 1989).

[26] M. de León, J.C. Marrero and D. Martín de Diego. Mechanical systems with non-linear constraints. Int. J. Theor. Phys. 36 (1997), 973-989.

[27] M. DE León and D. Martín De Diego. On the geometry of non-holonomic lagrangian systems. J. Math. Phys. 37 (1996), 3389-3414.

[28] A.D. LEWIS. Affine connections and distributions with applications to nonholonomic mechanics. Rep. Math. Phys. 42 (1998), 135-164.

[29] A.D. Lewis and R.M. Murray. Configuration controllability of simple mechanical control systems. SIAM J. Control Optim. 35 (1997), 766-790.

[30] C.-M. Marle. Reduction of Constrained mechanical systems and stability of relative equilibria. Commun. Math. Phys. 174 (1995), 295-318.

[31] J.E. Marsden, R. Montgomery and T. Ratiu. Reduction, symmetry, and phases in mechanics. Mem. Amer. Math. Soc., Vol. 436 (AMS, Providence, 1990).

[32] E. Massa and E. Pagani. A new look at classical mechanics of constrained systems. Ann. Inst. H. Poincaré (Phys. Théor.) 66 (1997), 1-36.

[33] J. Neimark and N. Fufaev. Dynamics of Nonholonomic Systems. Transactions of Mathematical Monographs, Vol. 33 (AMS, Providence, 1972). 
[34] J. Ostrowski. Geometric Perspectives on the Mechanics and Control of Undulatory Locomotion. PhD Thesis (California Institute of Technology, 1995)

[35] A. Shapere and F. WilczeK. Geometry of self-propulsion at low Reynolds number. J. Fluid. Mech. 198 (1989), 557-585.

[36] S.V. Stanchenko. Nonholonomic Chaplygin systems. Prikl. Mat. Mehk. 53 (1989), 16-23 (in Russian).

[37] D. Saunders, F. Cantrijn and W. Sarlet. Regularity aspects and Hamiltonization of non-holonomic systems. J. Phys. A: Math. Gen. 32 (1999), 6869-6890.

[38] J.Z. Synge. Geodesics in nonholonomic geometry. Math. Annalen 99 (1928), 738-751.

[39] A.J. VAn Der Schaft and B.M. Maschke. On the Hamiltonian formulation of nonholonomic mechanical systems. Rep. Math. Phys. 34 (1994), 225-233.

[40] A.M. Vershik and L.D. Fadeev. Lagrangian mechanics in invariant form. Sel. Math. Sov. 1 (1981), 339-350.

[41] A.P. Veselov and L.E. Veselova. Flows on Lie groups with nonholonomic constraint and integrable nonhamiltonian systems. Funkt. Anal. Prilozh. 20 (1986), 65-66 (in Russian), Funct. Anal. Appl. 20 (1986), 308-309 (Engl. transl.)

[42] L.S. Wang and P.S. Krishnaprasad. Gyroscopic control and stabilization. J. Nonlinear Sci. 2 (1992), 367-415.

[43] R. YAng. Nonholonomic Geometry, Mechanics and Control. PhD Thesis (Systems Research Institute, Univ. of Maryland, 1992). 